\title{
Synthesis of new functionalized aziridine-2- and azetidine-3-carboxylic acid derivatives of potential interest for biological and foldameric applications
}

\author{
Asta Žukauskaitè, ${ }^{\mathrm{a}, \mathrm{b}}$ Sven Mangelinckx, ${ }^{\mathrm{a}, \dagger}$ Vida Buinauskaitè, ${ }^{\mathrm{b}}$ Algirdas Šačkus ${ }^{\mathrm{b}}$ \\ and Norbert De Kimpe ${ }^{\mathrm{a}, *}$ \\ ${ }^{a}$ Department of Sustainable Organic Chemistry and Technology, Faculty of \\ Bioscience Engineering, Ghent University, Coupure Links 653, B-9000 Ghent, \\ Belgium \\ ${ }^{b}$ Institute of Synthetic Chemistry, Kaunas University of Technology, LT-50270 \\ Kaunas, Lithuania \\ ${ }^{\dagger}$ Postdoctoral Fellow of the Research Foundation-Flanders (FWO) \\ norbert.dekimpe@ugent.be
}

\begin{abstract}
A short synthesis of alkyl 2-(bromomethyl)aziridine-2-carboxylates and alkyl 3bromoazetidine-3-carboxylates was developed involving amination, bromination and base-induced cyclization of alkyl 2-(bromomethyl)acrylates. The aziridines are the kinetically favored cyclization products and could be transformed into 3-bromoazetidine-3-carboxylic acid derivatives via thermal isomerization. The new small-membered azaheterocyclic $\alpha$ - and $\beta$-amino acid derivatives contain a bromo-substituted carbon center as a useful moiety for functionalization. Transformation of these functionalized azaheterocycles via nucleophilic substitution with carbon, sulfur, oxygen and nitrogen nucleophiles and via elaboration of the amino and carboxyl group provided a broad range of new conformationally constrained aziridine-2- and azetidine-3carboxylic acid derivatives which are of interest from a biological point-of-view as well as for applications in the field of foldamers.
\end{abstract}

Keywords Aziridine-2-carboxylic acids, Azetidine-3-carboxylic acids, $\beta$-Amino acids, $\alpha$-Amino acids, Conformational constraint

\section{Introduction}

Aziridine-2-carboxylic acid derivatives are biologically important constrained amino acid derivatives and versatile building blocks in the preparation of proteinogenic and non-proteinogenic amino acids and biologically active nitrogen-containing compounds (Cardillo et al. 2003, 2006; Lee and Ha 2003; 
Lucarini and Tomasini 2001; Ide et al. 2005; Vicik et al. 2006). The latter is nicely exemplified by the ring opening of $(2 R)-N$-(acetyl)aziridine-2-carboxylates $\mathbf{1}$ in the synthesis of lacosamide (2, Vimpat ${ }^{\circledR}$; UCB), approved in 2008 for the adjunctive treatment of partial onset seizures in patients with epilepsy (Figure 1) (Morieux et al. 2008; Hughes 2009b). The different isomers of 2-(4-amino-4carboxybutyl)aziridine-2-carboxylate $\mathbf{3}$ (AziDAP) are irreversible inhibitors and substrate mimics of diaminopimelate (DAP) epimerase, a key enzyme for the biosynthesis of lysine in plants (Pillai et al. 2006, 2009). In view of the analogy with constrained $\beta^{2,2}$-amino acids such as $\beta^{2}$-HAib 5, $3{ }_{14}$-helix- and sheetbreaking in $\beta$-peptides (Seebach and Gardiner 2008; Cheng et al. 2001), and 1(aminomethyl)cyclopropanecarboxylic acid 6, which forms $\beta$-oligopeptides with a ribbon- or stair-like structure (Abele et al. 1999), the unknown 2(aminomethyl)aziridine-2-carboxylic acids $\mathbf{4}$ are of significant interest as potential foldameric building blocks. Alternatively, derivatives of azetidine-3-carboxylic acid disclose gametocidal activity (Zhang et al. 2004; Verbrugge and De Waal 1989; Orr and Clifford 1984; Devlin 1981), have found application as $\beta$-proline analogues (Mazzini et al. 1997), and have been used for the preparation of a variety of pharmaceutically active compounds (Miller et al. 2003). More specifically, a 3-aminoazetidine-3-carboxylic acid derivative has been incorporated in CE-178,253 (7, Pfizer), a $\mathrm{CB}_{1}$ antagonist for the treatment of obesity (Brandt et al. 2009). Furthermore, 3-aminoazetidine-3-carboxylic acid derivatives $\mathbf{8}$ also represent interesting conformationally constrained functionalized $C^{\alpha}$-tetrasubstituted $\alpha$-amino acids, which have not been studied in the foldameric field. Related $C^{\alpha}$-tetrasubstituted $\alpha$-amino acids such as Aib (9) and 1-aminocyclobutane-1-carboxylic acid $\left(\mathbf{1 0}, \mathrm{Ac}_{4} \mathrm{c}\right)$ are known to form short peptides with a conformational preference for $\beta$-turns and $3_{10}$-helices (Toniolo et al. 2006; Maity and König 2008; Soloshonok and Sorochinsky 2010).

In view of the importance of the aforementioned classes of amino acids, the development of pathways to new three- and four-membered azaheterocyclic $\alpha$ and $\beta$-amino acid derivatives is a challenging research field in modern synthetic chemistry at the biological interface (Hughes 2009a; Kiss and Fülöp 2010; Fülöp et al. 2006; Kuhl et al. 2005; Miller and Nguyen 2005; Cativiela and Ordóñez 2009; Komarov et al. 2004; Cowell et al. 2004; Gelmi and Pocar 2003; Park and Kurth 2002). 

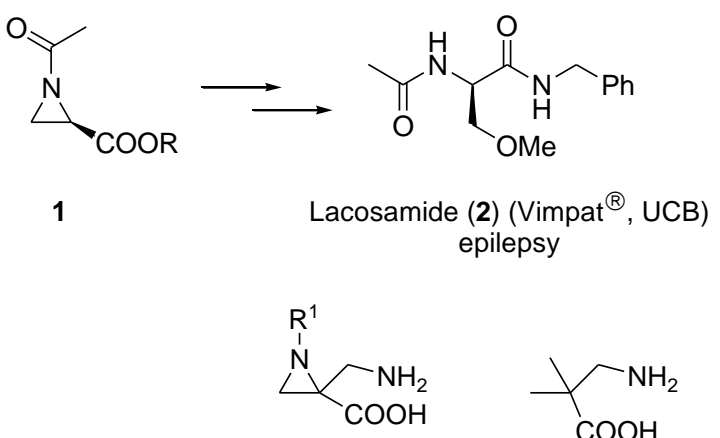

4

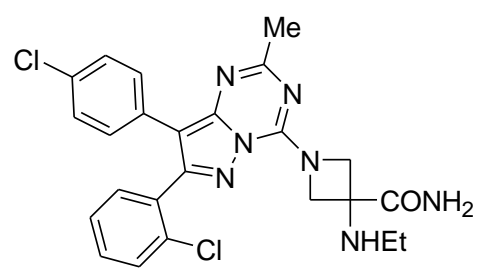

CE-178,253 (7) (Pfizer)

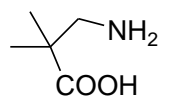

$\beta^{2}$-HAib (5)
8<smiles>CC(N)C(CCCC(N)C(=O)O)CC(=O)O</smiles>

LL-Azi-DAP (3)<smiles>CC(CCCC(N)C(=O)O)CC(=O)O</smiles>

DL-Azi-DAP (3)

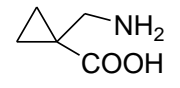

6<smiles>CC(N)(C(=O)O)C(=O)O</smiles>

Aib (9)

$\mathrm{AC}_{4} \mathrm{C}(\mathbf{1 0})$

Fig. 1 Several aziridine-2- and azetidine-3-carboxylic acid derivatives and related compounds with biological or foldameric applications

Following a preliminary communication (Mangelinckx et al. 2008), in the present paper, in-depth results are described on the synthesis and study of the reactivity of 2-(bromomethyl)aziridine-2-carboxylic esters and 3-bromoazetidine-3-carboxylic esters, the structures of which incorporate the synthetically important 2(halomethyl)aziridine (Abbaspour Tehrani et al. 2002; De Smaele et al. 1998; De Kimpe et al. 1997; D'hooghe et al. 2006a; Mangelinckx et al. 2009; Vervisch et al. 2010), or 3-haloazetidine structural motif (Van Driessche et al. 2006; Van Brabandt et al. 2009). The bromo-substituted carbon atom can be advantageously used as a moiety for functionalization. Allylamines have proven to be suitable substrates for the synthesis of 2-(bromomethyl)aziridines and/or 3bromoazetidines via bromination of the $\mathrm{C}-\mathrm{C}$ double bond to the corresponding $\beta, \gamma$-dibromo amines followed by intramolecular ring closure (Abbaspour Tehrani et al. 2002; De Smaele et al. 1998; De Kimpe et al. 1997; Hayashi et al. 2004; Gensler 1948), or via electrophile-induced bromocyclization (Robin and Rousseau 2000, 2002). This ring closure usually has high selectivity towards the formation of aziridines instead of azetidines. 


\section{Material and methods}

Reagents and solvents were purchased from commercial suppliers and used without further purification, unless indicated otherwise. Dichloromethane was distilled from calcium hydride before use. The purification of reaction mixtures was performed through flash chromatography using a glass column with silica gel (0.035-0.070 nm, pore diameter ca. $6 \mathrm{~nm})$ or by preparative thin layer chromatography on silica gel (silica gel GF preparative layer with UV 254, 2 $\mathrm{mm}$ ). Solvent systems were evaluated via TLC analysis (silica gel $2-25 \mu \mathrm{m}$, thickness $0.25 \mathrm{~mm}$, medium pore diameter $60 \AA$ with UV 254). ${ }^{1} \mathrm{H}$ NMR (300 $\mathrm{MHz}),{ }^{13} \mathrm{C}$ NMR $(75 \mathrm{MHz})$ spectra were recorded on a JEOL ECLIPSE FT 300 NMR spectrometer at room temperature. The compounds were dissolved in deuterated solvents as indicated for each compound. Tetramethylsilane (TMS) was used as an internal standard. IR spectra were recorded on a Perkin-Elmer Spectrum BX FT-IR spectrometer in neat form with an ATR (Attenuated Total Reflectance) accessory. Alternatively, IR spectra were recorded on a PerkinElmer Spectrum One spectrometer. For liquid samples the spectra were collected by preparing a thin film of compound between two sodium chloride plates. Mass spectra were recorded on an Agilent 1100 series mass spectrometer using either a direct inlet system (electron spray, 4000V) or LC-MS coupling (UV detector).

Synthesis of alkyl 2-[(alkylamino)methyl]acrylates 12a-d

As a representative example, the synthesis of methyl 2-[(tertpentylamino)methyl]acrylate (12d) is described here. To a solution of tertpentylamine (175 mg, $2 \mathrm{mmol}$ ) and triethylamine (210 mg, $2.08 \mathrm{mmol})$ in $\mathrm{CH}_{2} \mathrm{Cl}_{2}$ $(10 \mathrm{~mL})$, a solution of methyl 2-(bromomethyl)acrylate (11b) $(358 \mathrm{mg}, 2 \mathrm{mmol})$ in $\mathrm{CH}_{2} \mathrm{Cl}_{2}(5 \mathrm{~mL})$ was added dropwise while stirring at $0{ }^{\circ} \mathrm{C}$. After the reaction was stirred for $30 \mathrm{~min}$ at $0{ }^{\circ} \mathrm{C}$, water $(20 \mathrm{~mL})$ was added to the reaction mixture, which was extracted with $\mathrm{CH}_{2} \mathrm{Cl}_{2}(3 \times 20 \mathrm{~mL})$. Drying $\left(\mathrm{MgSO}_{4}\right)$, filtration and evaporation of the solvent under reduced pressure afforded methyl 2-[(tertpentylamino)methyl]acrylate (12d) in $98 \%$ yield which was used without further purification. 
Yellow oil, yield 97\%, $\mathrm{R}_{f}=0.50$ (petroleum ether/EtOAc 7/3). The ${ }^{1} \mathrm{H}$ NMR data were in good agreement with reported data (Habaue et al. 1997). ${ }^{1} \mathrm{H}$ NMR (300 $\left.\mathrm{MHz}, \mathrm{CDCl}_{3}\right): \delta 1.14\left(9 \mathrm{H}, \mathrm{s}, \mathrm{C}\left(\mathrm{CH}_{3}\right)_{3}\right), 1.31\left(3 \mathrm{H}, \mathrm{t}, J=7.2 \mathrm{~Hz}, \mathrm{CH}_{2} \mathrm{CH}_{3}\right), 3.40$ $\left(2 \mathrm{H}, \mathrm{s}, \mathrm{NCH}_{2}\right), 4.22\left(2 \mathrm{H}, \mathrm{q}, J=7.2 \mathrm{~Hz}, \mathrm{CH}_{2} \mathrm{CH}_{3}\right), 5.78(1 \mathrm{H}, \mathrm{dt}, J=1.3 \mathrm{~Hz}, 1.5$ $\mathrm{Hz}, \mathrm{C}=\mathrm{CH}(\mathrm{H})), 6.23(1 \mathrm{H}, \mathrm{s}, \mathrm{C}=\mathrm{CH}(H))$. Anal. Calcd for $\mathrm{C}_{10} \mathrm{H}_{19} \mathrm{NO}_{2}(\%): \mathrm{C}$, 64.83; H, 10.34; N, 7.56. Found (\%): C, 64.58; H, 10.56; N, 7.41.

Ethyl 2-[(tert-pentylamino)methyl]acrylate $(\mathbf{1 2 b})$

Colorless oil, yield 91\%, $\mathrm{R}_{f}=0.51$ (petroleum ether/EtOAc 7/3). ${ }^{1} \mathrm{H}$ NMR (300 $\left.\mathrm{MHz}, \mathrm{CDCl}_{3}\right): \delta 0.86\left(3 \mathrm{H}, \mathrm{t}, J=7.4 \mathrm{~Hz}, \mathrm{CCH}_{2} \mathrm{CH}_{3}\right), 1.06\left(6 \mathrm{H}, \mathrm{s}, \mathrm{C}\left(\mathrm{CH}_{3}\right)_{2}\right), 1.31$ $\left(3 \mathrm{H}, \mathrm{t}, J=7.2 \mathrm{~Hz}, \mathrm{OCH}_{2} \mathrm{CH}_{3}\right), 1.44\left(2 \mathrm{H}, \mathrm{q}, J=7.4 \mathrm{~Hz}, \mathrm{CCH}_{2} \mathrm{CH}_{3}\right), 3.34(2 \mathrm{H}, \mathrm{s}$, $\left.\mathrm{NCH}_{2}\right), 4.22\left(2 \mathrm{H}, \mathrm{q}, J=7.2 \mathrm{~Hz}, \mathrm{OCH}_{2} \mathrm{CH}_{3}\right), 5.79(1 \mathrm{H}, \mathrm{dt}, J=1.4 \mathrm{~Hz}, 1.4 \mathrm{~Hz}$, $\mathrm{C}=\mathrm{C} H(\mathrm{H})), 6.23(1 \mathrm{H}, \mathrm{d}, J=0.8 \mathrm{~Hz}, \mathrm{C}=\mathrm{CH}(H)) .{ }^{13} \mathrm{C} \mathrm{NMR}\left(75 \mathrm{MHz}, \mathrm{CDCl}_{3}\right): \delta$ $8.2,14.2,26.6,33.1,43.2,52.7,60.6,125.4,140.0,166.9 . \mathrm{IR}\left(\mathrm{NaCl}, \mathrm{cm}^{-1}\right) v_{\mathrm{NH}}=$ $3328, v_{\mathrm{C}=\mathrm{O}}=1717, v_{\mathrm{C}=\mathrm{C}}=1635$. MS (ES, pos. mode): $\mathrm{m} / z(\%): 200\left(\mathrm{M}+\mathrm{H}^{+}, 100\right)$. Anal. Calcd for $\mathrm{C}_{11} \mathrm{H}_{21} \mathrm{NO}_{2}(\%)$ : C, 66.29; H, 10.62; N, 7.03. Found (\%): C, 66.12; H, 10.37; N, 7.22.

Methyl 2-[(tert-butylamino)methyl]acrylate (12c)

Colorless oil, yield 94\%, $\mathrm{R}_{f}=0.48$ (petroleum ether/EtOAc 7/3). ${ }^{1} \mathrm{H}$ NMR (300 $\left.\mathrm{MHz}, \mathrm{CDCl}_{3}\right): \delta 1.14\left(9 \mathrm{H}, \mathrm{s}, \mathrm{C}\left(\mathrm{CH}_{3}\right)_{3}\right), 3.41\left(2 \mathrm{H}\right.$, br s, $\left.\mathrm{NCH}_{2}\right), 3.77(3 \mathrm{H}, \mathrm{s}$, $\left.\mathrm{COOCH}_{3}\right), 5.80(1 \mathrm{H}, \mathrm{dt}, J=1.5 \mathrm{~Hz}, 1.7 \mathrm{~Hz}, \mathrm{C}=\mathrm{CH}(\mathrm{H})), 6.23(1 \mathrm{H}, \mathrm{d}, J=1.1 \mathrm{~Hz}$, $\mathrm{C}=\mathrm{CH}(H)) .{ }^{13} \mathrm{C} \mathrm{NMR}\left(75 \mathrm{MHz}, \mathrm{CDCl}_{3}\right): \delta 29.1,43.8,50.6,51.8,125.5,139.9$, 167.4. IR $\left(\mathrm{NaCl}, \mathrm{cm}^{-1}\right): v_{\mathrm{NH}}=3327, v_{\mathrm{C}=\mathrm{O}}=1719, v_{\mathrm{C}=\mathrm{C}}=1635$. MS (ES, pos. mode): $m / z(\%): 172\left(\mathrm{M}+\mathrm{H}^{+}, 100\right)$. Anal. Calcd for $\mathrm{C}_{9} \mathrm{H}_{17} \mathrm{NO}_{2}(\%): \mathrm{C}, 63.13 ; \mathrm{H}$, $10.01 ; \mathrm{N}, 8.18$. Found (\%): C, 63.35; H, 10.34; N, 7.96.

Methyl 2-[(tert-pentylamino)methyl]acrylate $(\mathbf{1 2 d})$ 
Yellow oil, yield 98\%, $\mathrm{R}_{f}=0.50$ (petroleum ether/EtOAc 7/3). ${ }^{1} \mathrm{H}$ NMR (300 $\left.\mathrm{MHz} \mathrm{CDCl}_{3}\right): \delta 0.86\left(3 \mathrm{H}, \mathrm{t}, J=7.4 \mathrm{~Hz}, \mathrm{CH}_{2} \mathrm{CH}_{3}\right), 1.07\left(6 \mathrm{H}, \mathrm{s}, \mathrm{C}\left(\mathrm{CH}_{3}\right)_{2}\right), 1.44$ $\left(2 \mathrm{H}, \mathrm{q}, J=7.4 \mathrm{~Hz}, \mathrm{CH}_{2} \mathrm{CH}_{3}\right), 3.35\left(2 \mathrm{H}, \mathrm{s}, \mathrm{CH}_{2} \mathrm{NH}\right), 3.77\left(3 \mathrm{H}, \mathrm{s}, \mathrm{OCH}_{3}\right), 5.81(1 \mathrm{H}$, $\mathrm{dt}, J=1.7 \mathrm{~Hz}, 1.4 \mathrm{~Hz}, \mathrm{C}=\mathrm{CH}(\mathrm{H})), 6.23(1 \mathrm{H}, \mathrm{d}, J=1.4 \mathrm{~Hz}, \mathrm{C}=\mathrm{CH}(H)) .{ }^{13} \mathrm{C} \mathrm{NMR}$ $\left(75 \mathrm{MHz}, \mathrm{CDCl}_{3}\right): \delta 8.3,26.7,33.2,43.2,51.9,52.8,125.7,139.9,167.5 . \mathrm{IR}$ $\left(\right.$ neat, $\left.\mathrm{cm}^{-1}\right): v_{\mathrm{NH}}=3336($ weak $), v_{\mathrm{C}=\mathrm{O}}=1718, v_{\mathrm{C}=\mathrm{C}}=1634$. MS (ES, pos. mode): $m / z$ (\%): $186\left(\mathrm{M}+\mathrm{H}^{+}, 100\right)$. Anal. Calcd for $\mathrm{C}_{10} \mathrm{H}_{19} \mathrm{NO}_{2}(\%): \mathrm{C}, 64.83 ; \mathrm{H}, 10.34$; N, 7.56. Found (\%): C, 64.60; H, 10.41; N, 7.34.

Bromination of alkyl 2-[(alkylamino)methyl]acrylates 12a-d

As a representative example, the synthesis of methyl 2-[(tertpentylamino)methyl]-2,3-dibromopropanoate (13d) is described here. To a solution of methyl 2-[(tert-pentylamino)methyl]acrylate (12d) $(330 \mathrm{mg}, 1.78$ mmol) in $\mathrm{CH}_{2} \mathrm{Cl}_{2}(30 \mathrm{~mL})$, a $48 \%$ aqueous solution of hydrobromic acid (332 $\mathrm{mg}$, $1.96 \mathrm{mmol}$ ) was added dropwise at $0{ }^{\circ} \mathrm{C}$ and the reaction mixture was stirred for 30 min. Then, a solution of $\mathrm{Br}_{2}(285 \mathrm{mg}, 1.78 \mathrm{mmol})$ in $\mathrm{CH}_{2} \mathrm{Cl}_{2}(15 \mathrm{~mL})$ was added dropwise and the reaction mixture was left stirring upon warming to room temperature for $5 \mathrm{~h}$. Subsequently, the resulting reaction mixture was neutralised by pouring into saturated $\mathrm{NaHCO}_{3}$ solution and extracted with EtOAc $(3 \times 20$ $\mathrm{mL})$. Drying $\left(\mathrm{MgSO}_{4}\right)$, filtration, evaporation of the solvent under reduced pressure and purification by flash chromatography on silica gel (petroleum ether/EtOAc 9/1) afforded dibromo ester 13d in 97\% yield.

Ethyl 2-[(tert-butylamino)methyl]-2,3-dibromopropanoate (13a)

Colorless oil, yield 98\%, $\mathrm{R}_{f}=0.50$ petroleum ether/EtOAc 9/1). ${ }^{1} \mathrm{H}$ NMR (300 $\left.\mathrm{MHz}, \mathrm{CDCl}_{3}\right): \delta 1.16\left(9 \mathrm{H}, \mathrm{s}, \mathrm{C}\left(\mathrm{CH}_{3}\right)_{3}\right), 1.34\left(3 \mathrm{H}, \mathrm{t}, J=7.2 \mathrm{~Hz}, \mathrm{CH}_{2} \mathrm{CH}_{3}\right), 3.24$ $(1 \mathrm{H}, \mathrm{d}, J=13.8 \mathrm{~Hz}, \mathrm{NCH}(\mathrm{H})), 3.30(1 \mathrm{H}, \mathrm{d}, J=13.8 \mathrm{~Hz}, \mathrm{NCH}(H)), 4.08(1 \mathrm{H}, \mathrm{d}, J$ $=9.9 \mathrm{~Hz}, \mathrm{CH}(\mathrm{H}) \mathrm{Br}), 4.23(1 \mathrm{H}, \mathrm{d}, J=9.9 \mathrm{~Hz}, \mathrm{CH}(H) \mathrm{Br}), 4.22-4.39(2 \mathrm{H}, \mathrm{m}$, $\left.\mathrm{CH}_{2} \mathrm{CH}_{3}\right) .{ }^{13} \mathrm{C} \mathrm{NMR}\left(75 \mathrm{MHz}, \mathrm{CDCl}_{3}\right): \delta 13.9,28.5,34.6,46.6,54.0,59.8,63.0$, 168.0. IR $\left(\mathrm{NaCl}, \mathrm{cm}^{-1}\right): v_{\mathrm{NH}}=3327, v_{\mathrm{C}=\mathrm{O}}=1739$. MS (ES, pos. mode): $\mathrm{m} / \mathrm{z}(\%)$ : 344/346/348 (M+H' $\left.{ }^{+}, 100\right)$. Anal. Calcd for $\mathrm{C}_{10} \mathrm{H}_{19} \mathrm{Br}_{2} \mathrm{NO}_{2}(\%)$ : C, 34.81; H, 5.55; N, 4.06. Found (\%): C, 34.48; H, 5.32; N, 3.84. 
Colorless oil, yield 97\%, $\mathrm{R}_{f}=0.71\left(\mathrm{CH}_{2} \mathrm{Cl}_{2}\right) .{ }^{1} \mathrm{H} \mathrm{NMR}\left(300 \mathrm{MHz}, \mathrm{CDCl}_{3}\right): \delta 0.86$ $\left(3 \mathrm{H}, \mathrm{t}, J=7.4 \mathrm{~Hz}, \mathrm{CCH}_{2} \mathrm{CH}_{3}\right), 1.04\left(3 \mathrm{H}, \mathrm{s}, \mathrm{CCH}_{3}\left(\mathrm{CH}_{3}\right)\right), 1.05(3 \mathrm{H}, \mathrm{s}$, $\left.\mathrm{CCH}_{3}\left(\mathrm{CH}_{3}\right)\right), 1.34\left(3 \mathrm{H}, \mathrm{t}, J=7.2 \mathrm{~Hz}, \mathrm{OCH}_{2} \mathrm{CH}_{3}\right), 1.40(2 \mathrm{H}, \mathrm{q}, J=7.3 \mathrm{~Hz}$, $\left.\mathrm{CCH}_{2} \mathrm{CH}_{3}\right), 3.15(1 \mathrm{H}, \mathrm{d}, J=13.8 \mathrm{~Hz}, \mathrm{NCH}(H)), 3.20(1 \mathrm{H}, \mathrm{d}, J=13.8 \mathrm{~Hz}$, $\mathrm{NCH}(\mathrm{H})), 4.06(1 \mathrm{H}, \mathrm{d}, J=9.6 \mathrm{~Hz}, \mathrm{CBrH}(H)), 4.22(1 \mathrm{H}, \mathrm{d}, J=9.6 \mathrm{~Hz}, \mathrm{CBr} H(\mathrm{H}))$, $4.28(1 \mathrm{H}, \mathrm{dq}, \mathrm{J}=7.2 \mathrm{~Hz}, 10.7 \mathrm{~Hz}, \mathrm{OCH}(H)), 4.32(1 \mathrm{H}, \mathrm{dq}, J=7.2 \mathrm{~Hz}, 10.7 \mathrm{~Hz}$, $\mathrm{OCH}(\mathrm{H})) .{ }^{13} \mathrm{C} \mathrm{NMR}\left(75 \mathrm{MHz}, \mathrm{CDCl}_{3}\right): \delta 8.2,13.9,26.8,27.0,33.6,34.4,45.9$, 52.4, 62.4, 62.7, 168.2. IR $\left(\mathrm{NaCl}, \mathrm{cm}^{-1}\right): v_{\mathrm{NH}}=3327$ (weak), $v_{\mathrm{C}=\mathrm{O}}=1742 . \mathrm{MS}$ (ES, pos. mode): $\mathrm{m} / \mathrm{z}(\%): 358 / 360 / 362\left(\mathrm{M}+\mathrm{H}^{+}, 100\right)$. Anal. Calcd for $\mathrm{C}_{11} \mathrm{H}_{21} \mathrm{Br}_{2} \mathrm{NO}_{2}$ (\%): C, 36.79; H, 5.89; N, 3.90. Found (\%): C, 36.62; H, 6.12; N, 3.81 .

Methyl 2-[(tert-butylamino)methyl]-2,3-dibromopropanoate (13c)

Yellow oil, yield 90\%, $\mathrm{R}_{f}=0.53$ (petroleum ether/EtOAc 9/1). Spectroscopically characterized as the hydrobromide before neutralization. ${ }^{1} \mathrm{H}$ NMR $(300 \mathrm{MHz}$, DMSO-d $\left.\mathrm{d}_{6}\right): \delta 1.37\left(9 \mathrm{H}, \mathrm{s}, \mathrm{C}\left(\mathrm{CH}_{3}\right)_{3}\right), 3.69\left(2 \mathrm{H}\right.$, br s, $\left.\mathrm{NCH}_{2}\right), 3.84\left(3 \mathrm{H}, \mathrm{s}, \mathrm{OCH}_{3}\right)$, $4.27(1 \mathrm{H}, \mathrm{d}, J=11.0 \mathrm{~Hz}, \operatorname{BrCH}(\mathrm{H})), 4.41(1 \mathrm{H}, \mathrm{d}, J=10.7 \mathrm{~Hz}, \operatorname{BrCH}(H)), 8.58$ and $8.65(2 \mathrm{H}, 2 \times \mathrm{br} \mathrm{s}, \mathrm{NH} \cdot \mathrm{HBr}) .{ }^{13} \mathrm{C} \mathrm{NMR}\left(75 \mathrm{MHz}, \mathrm{DMSO}_{-} \mathrm{d}_{6}\right): \delta 24.8,35.3$, 46.2, 54.1, 55.6, 59.0, 166.7. IR $\left(\mathrm{NaCl}, \mathrm{cm}^{-1}\right): v_{\mathrm{NH}}=3445, v_{\mathrm{C}=\mathrm{O}}=1740 . \mathrm{MS}(\mathrm{ES}$, pos. mode): $\mathrm{m} / \mathrm{z}(\%)$ : 330/332/334 $\left(\mathrm{M}-\mathrm{HBr}+\mathrm{H}^{+}, 100\right)$. Anal. Calcd for $\mathrm{C}_{9} \mathrm{H}_{17} \mathrm{Br}_{2} \mathrm{NO}_{2}$ (\%): C, 32.65; H, 5.18; N, 4.23. Found (\%): C, 32.29; H, 5.36; N, 4.07 .

Methyl 2-[(tert-pentylamino)methyl]-2,3-dibromopropanoate (13d)

Yellow oil, yield 97\%, $\mathrm{R}_{f}=0.50$ (petroleum ether/EtOAc 9/1). ${ }^{1} \mathrm{H}$ NMR (300 $\left.\mathrm{MHz}, \mathrm{CDCl}_{3}\right): \delta 0.86\left(3 \mathrm{H}, \mathrm{t}, J=7.4 \mathrm{~Hz}, \mathrm{CH}_{2} \mathrm{CH}_{3}\right), 1.04\left(3 \mathrm{H}, \mathrm{s}, \mathrm{CH}_{3} \mathrm{CCH}_{3}\right), 1.05$ $\left(3 \mathrm{H}, \mathrm{s}, \mathrm{CH}_{3} \mathrm{CCH}_{3}\right), 1.40\left(2 \mathrm{H}, \mathrm{q}, J=7.4 \mathrm{~Hz}, \mathrm{CH}_{2} \mathrm{CH}_{3}\right), 1.56(1 \mathrm{H}$, br s, NH), 3.16 $(1 \mathrm{H}, \mathrm{d}, J=13.5 \mathrm{~Hz}, \mathrm{NHCH}(\mathrm{H})), 3.20(1 \mathrm{H}, \mathrm{d}, J=13.8 \mathrm{~Hz}, \mathrm{NHCH}(H)), 3.84(3 \mathrm{H}$, s, $\left.\mathrm{OCH}_{3}\right), 4.06(1 \mathrm{H}, \mathrm{d}, J=9.6 \mathrm{~Hz}, \mathrm{CH}(\mathrm{H}) \mathrm{Br}), 4.22(1 \mathrm{H}, \mathrm{d}, J=9.6 \mathrm{~Hz}, \mathrm{CH}(H) \mathrm{Br})$. 
${ }^{13} \mathrm{C}$ NMR (75 MHz, $\mathrm{CDCl}_{3}$ ): $\delta$ 8.4, 26.9, 27.0, 33.7, 34.5, 46.1, 52.5, 53.4, 62.6, 168.9. MS (ES, pos. mode): $m / z(\%): 344 / 346 / 348\left(\mathrm{M}+\mathrm{H}^{+}, 100\right)$. Anal. Calcd for $\mathrm{C}_{10} \mathrm{H}_{19} \mathrm{Br}_{2} \mathrm{NO}_{2}$ (\%): C, 34.81; H, 5.55; N, 4.06. Found (\%): C, 34.69; H, 5.75; N, 3.80 .

Synthesis of alkyl 2-(bromomethyl)aziridine-2-carboxylates 14a-d and alkyl 3bromoazetidine-3-carboxylates 15a-d

As a representative example, the synthesis of methyl 1-tert-pentyl-2(bromomethyl)aziridine-2-carboxylate (14d) and methyl 1-tert-pentyl-3bromoazetidine-3-carboxylate (15d) is described here. To a solution of methyl 2[(tert-pentylamino)methyl]-2,3-dibromopropanoate (13d) $(618 \mathrm{mg}, 1.79 \mathrm{mmol})$ in $\mathrm{CH}_{3} \mathrm{CN}(30 \mathrm{~mL})$ was added powdered $\mathrm{K}_{2} \mathrm{CO}_{3}(371 \mathrm{mg}, 2.69 \mathrm{mmol})$ and the reaction mixture was stirred at $60{ }^{\circ} \mathrm{C}$ for $18 \mathrm{~h}$. Then the solvent was removed under reduced pressure and $\mathrm{Et}_{2} \mathrm{O}(30 \mathrm{~mL})$ was added. The resulting solution was filtered and the filter cake was washed with small portions of $\mathrm{Et}_{2} \mathrm{O}$. Evaporation of the solvent under reduced pressure and purification by preparative TLC on silica gel (petroleum ether/Et $2 \mathrm{O} 7 / 3$ ) afforded aziridine 14d in 54\% yield and azetidine $\mathbf{1 5 d}$ in $26 \%$ yield.

\section{Ethyl 1-tert-butyl-2-(bromomethyl)aziridine-2-carboxylate (14a)}

Light yellow oil, yield 50\%, $\mathrm{R}_{f}=0.48$ (petroleum ether/Et $2 \mathrm{O} 7 / 3$ ). ${ }^{1} \mathrm{H}$ NMR (300 $\left.\mathrm{MHz}, \mathrm{CDCl}_{3}\right): \delta 1.08\left(9 \mathrm{H}, \mathrm{s},\left(\mathrm{CH}_{3}\right)_{3}\right), 1.33\left(3 \mathrm{H}, \mathrm{t}, J=7.2 \mathrm{~Hz}, \mathrm{CH}_{2} \mathrm{CH}_{3}\right), 1.83(1 \mathrm{H}$, $\mathrm{d}, J=1.1 \mathrm{~Hz}, \mathrm{NCH}(\mathrm{H})), 2.55(1 \mathrm{H}, \mathrm{dd}, J=1.1 \mathrm{~Hz}, 1.1 \mathrm{~Hz}, \mathrm{NCH}(H)), 3.03(1 \mathrm{H}, \mathrm{d}$, $J=9.9 \mathrm{~Hz}, \operatorname{BrCH}(\mathrm{H})), 4.05(1 \mathrm{H}, \mathrm{dd}, J=9.9 \mathrm{~Hz}, 1.1 \mathrm{~Hz}, \operatorname{BrCH}(H)), 4.21(1 \mathrm{H}, \mathrm{dq}$, $\left.J=10.7 \mathrm{~Hz}, 7.2 \mathrm{~Hz}, \mathrm{CH}(\mathrm{H}) \mathrm{CH}_{3}\right), 4.26(1 \mathrm{H}, \mathrm{dq}, J=11.0 \mathrm{~Hz}, 7.15 \mathrm{~Hz}$, $\left.\mathrm{CH}(H) \mathrm{CH}_{3}\right) .{ }^{13} \mathrm{C}$ NMR $\left(75 \mathrm{MHz}, \mathrm{CDCl}_{3}\right): \delta 14.1,28.2,33.6,37.0,45.2,55.1$, 61.8, 169.6. IR (neat, $\left.\mathrm{cm}^{-1}\right): v_{\mathrm{C}=\mathrm{O}}=1732$. MS (ES, pos. mode): $\mathrm{m} / z(\%): 264 / 266$ $\left(\mathrm{M}+\mathrm{H}^{+}, 100\right)$. Anal. Calcd for $\mathrm{C}_{10} \mathrm{H}_{18} \mathrm{BrNO}_{2}$ (\%): C, 45.47; H, 6.87; N, 5.30. Found (\%): C, 45.41; H, 6.98; N, 5.19. 
Yellow oil, yield 33\%, $\mathrm{R}_{f}=0.20$ (petroleum ether/Et $\mathrm{E}_{2} \mathrm{O}$ 7/3). ${ }^{1} \mathrm{H}$ NMR (300 MHz, $\left.\mathrm{CDCl}_{3}\right): \delta 0.96\left(9 \mathrm{H}, \mathrm{s}, \mathrm{C}\left(\mathrm{CH}_{3}\right)_{3}\right), 1.32\left(3 \mathrm{H}, \mathrm{t}, J=7.2 \mathrm{~Hz}, \mathrm{CH}_{2} \mathrm{CH}_{3}\right), 3.62(2 \mathrm{H}, \mathrm{d}, J$ $=9.9 \mathrm{~Hz}, \mathrm{CH}(\mathrm{H}) \mathrm{NCH}(\mathrm{H})), 3.93(2 \mathrm{H}, \mathrm{d}, J=9.9 \mathrm{~Hz}, \mathrm{CH}(H) \mathrm{NCH}(H)), 4.28(2 \mathrm{H}, \mathrm{q}$, $\left.J=7.2 \mathrm{~Hz}, \mathrm{CH}_{2} \mathrm{CH}_{3}\right) .{ }^{13} \mathrm{C} \mathrm{NMR}\left(75 \mathrm{MHz}, \mathrm{CDCl}_{3}\right): \delta 13.9,24.1,45.2,52.4,59.6$, 62.4, 170.4. IR (neat, $\left.\mathrm{cm}^{-1}\right): v_{\mathrm{C}=\mathrm{O}}=1739$. MS (ES, pos. mode): $\mathrm{m} / z(\%): 264 / 266$ $\left(\mathrm{M}+\mathrm{H}^{+}\right.$, 100). Anal. Calcd for $\mathrm{C}_{10} \mathrm{H}_{18} \mathrm{BrNO}_{2}$ (\%): C, 45.47; H, 6.87; N, 5.30. Found (\%): C, 45.38; H, 7.06; N, 5.41.

Ethyl 1-tert-pentyl-2-(bromomethyl)aziridine-2-carboxylate (14b)

Light yellow oil, yield 54\%, $\mathrm{R}_{f}=0.48$ (petroleum ether/Et ${ }_{2} \mathrm{O} 7 / 3$ ). ${ }^{1} \mathrm{H}$ NMR (300 $\left.\mathrm{MHz}, \mathrm{CDCl}_{3}\right): \delta 0.90\left(3 \mathrm{H}, \mathrm{t}, J=7.4 \mathrm{~Hz}, \mathrm{CCH}_{2} \mathrm{CH}_{3}\right), 0.95\left(3 \mathrm{H}, \mathrm{s}, \mathrm{CCH}_{3}\left(\mathrm{CH}_{3}\right)\right)$, $0.97\left(3 \mathrm{H}, \mathrm{s}, \mathrm{CCH}_{3}\left(\mathrm{CH}_{3}\right)\right), 1.33\left(3 \mathrm{H}, \mathrm{t}, J=7.2 \mathrm{~Hz}, \mathrm{OCH}_{2} \mathrm{CH}_{3}\right), 1.45(1 \mathrm{H}, \mathrm{qd}, J=$ $\left.7.3 \mathrm{~Hz}, 13.5 \mathrm{~Hz}, \mathrm{CCH}(\mathrm{H}) \mathrm{CH}_{3}\right), 1.49\left(1 \mathrm{H}, \mathrm{qd}, J=7.6 \mathrm{~Hz}, 13.6 \mathrm{~Hz}, \mathrm{CH}(H) \mathrm{CH}_{3}\right)$, $1.84(1 \mathrm{H}, \mathrm{d}, J=1.1 \mathrm{~Hz}, \mathrm{NCH}(\mathrm{H})), 2.59(1 \mathrm{H}, \mathrm{dd}, J=1.2 \mathrm{~Hz}, 1.2 \mathrm{~Hz}, \mathrm{NCH}(H))$, $3.03(1 \mathrm{H}, \mathrm{d}, J=9.9 \mathrm{~Hz}, \operatorname{BrCH}(\mathrm{H})), 4.07(1 \mathrm{H}, \mathrm{dd}, J=1.2 \mathrm{~Hz}, 10.0 \mathrm{~Hz}, \operatorname{BrCH}(H))$, $4.21(1 \mathrm{H}, \mathrm{dq}, J=7.0 \mathrm{~Hz}, 10.7 \mathrm{~Hz}, \mathrm{OCH}(\mathrm{H})), 4.26(1 \mathrm{H}, \mathrm{dq}, J=7.1 \mathrm{~Hz}, 10.6 \mathrm{~Hz}$, $\mathrm{OCH}(H)) .{ }^{13} \mathrm{C} \mathrm{NMR}\left(75 \mathrm{MHz}, \mathrm{CDCl}_{3}\right): \delta 8.7,14.0,24.0,24.4,33.7,36.4,37.0$, 44.4, 57.4, 61.6, 169.7. IR (neat, $\mathrm{cm}^{-1}$ ): $v_{\mathrm{C}=\mathrm{O}}=1736$. MS (ES, pos. mode): $\mathrm{m} / \mathrm{z}$ (\%): 278/280 (M+H+ $\mathrm{H}^{+}$, 100). Anal. Calcd for $\mathrm{C}_{11} \mathrm{H}_{20} \mathrm{BrNO}_{2}(\%)$ : C, 47.49; H, 7.25; N, 5.04. Found (\%): C, 47.24; H, 7.41; N, 4.82.

Ethyl 1-tert-pentyl-3-bromoazetidine-3-carboxylate (15b)

Yellow oil, yield 24\%, $\mathrm{R}_{f}=0.48$ (petroleum ether/EtOAc 9/1). ${ }^{1} \mathrm{H}$ NMR (300 $\left.\mathrm{MHz}, \mathrm{CDCl}_{3}\right): \delta 0.83\left(3 \mathrm{H}, \mathrm{t}, J=7.6 \mathrm{~Hz}, \mathrm{CCH}_{2} \mathrm{CH}_{3}\right), 0.88\left(6 \mathrm{H}, \mathrm{s}, \mathrm{C}\left(\mathrm{CH}_{3}\right)_{2}\right), 1.24$ $\left(2 \mathrm{H}, \mathrm{q}, J=7.4 \mathrm{~Hz}, \mathrm{CCH}_{2} \mathrm{CH}_{3}\right), 1.32\left(3 \mathrm{H}, \mathrm{t}, J=7.0 \mathrm{~Hz}, \mathrm{OCH}_{2} \mathrm{CH}_{3}\right), 3.60(2 \mathrm{H}, \mathrm{d}, J$ $=9.6 \mathrm{~Hz}, \mathrm{CH}(\mathrm{H}) \mathrm{NCH}(\mathrm{H})), 3.90(2 \mathrm{H}, \mathrm{d}, J=9.6 \mathrm{~Hz}, \mathrm{CH}(H) \mathrm{NCH}(H)), 4.28(2 \mathrm{H}, \mathrm{q}$, $\left.J=7.1 \mathrm{~Hz}, \mathrm{OCH}_{2}\right) .{ }^{13} \mathrm{C} \mathrm{NMR}\left(75 \mathrm{MHz}, \mathrm{CDCl}_{3}\right): \delta 8.5,13.9,20.1,31.5,45.5$, 54.7, 59.3, 62.2, 170.4. IR (neat, $\left.\mathrm{cm}^{-1}\right): v_{\mathrm{C}=\mathrm{O}}=1739$. MS (ES, pos. mode): $\mathrm{m} / \mathrm{z}$ (\%): 278/280 (M+H+ ${ }^{+}$, 90). Anal. Calcd for $\mathrm{C}_{11} \mathrm{H}_{20} \mathrm{BrNO}_{2}(\%)$ : C, 47.49; H, 7.25; N, 5.04. Found (\%): C, 47.41; H, 7.36; N, 4.84.

Methyl 1-tert-butyl-2-(bromomethyl)aziridine-2-carboxylate (14c) 
Light yellow oil, yield 44\%, $\mathrm{R}_{f}=0.45$ (petroleum ether/Et $\left.2 \mathrm{O} 7 / 3\right) .{ }^{1} \mathrm{H}$ NMR (300 $\left.\mathrm{MHz}, \mathrm{CDCl}_{3}\right): \delta 1.07\left(9 \mathrm{H}, \mathrm{s},\left(\mathrm{CH}_{3}\right)_{3}\right), 1.84(1 \mathrm{H}, \mathrm{d}, J=1.1 \mathrm{~Hz}, \mathrm{NCH}(\mathrm{H})), 2.55$ $(1 \mathrm{H}, \mathrm{dd}, J=1.1 \mathrm{~Hz}, 1.0 \mathrm{~Hz}, \mathrm{NCH}(H)), 3.06(1 \mathrm{H}, \mathrm{d}, J=9.9 \mathrm{~Hz}, \operatorname{BrCH}(\mathrm{H})), 3.78$ $\left(3 \mathrm{H}, \mathrm{s}, \mathrm{OCH}_{3}\right), 4.05(1 \mathrm{H}, \mathrm{dd}, J=10.2 \mathrm{~Hz}, 1.1 \mathrm{~Hz}, \mathrm{BrCH}(H)) .{ }^{13} \mathrm{C} \mathrm{NMR}(75 \mathrm{MHz}$, $\left.\mathrm{CDCl}_{3}\right): \delta 28.2,33.6,36.7,45.0,52.7,55.1,170.2$. IR (neat, $\mathrm{cm}^{-1}$ ): $v_{\mathrm{C}=\mathrm{O}}=1732$.

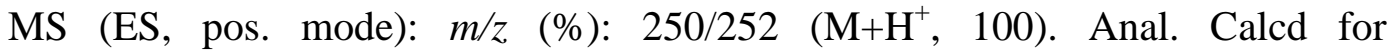
$\mathrm{C}_{9} \mathrm{H}_{16} \mathrm{BrNO}_{2}(\%): \mathrm{C}, 43.22 ; \mathrm{H}, 6.45 ; \mathrm{N}, 5.60$. Found (\%): C, 43.06; H, 6.69; N, 5.34 .

Methyl 1-tert-butyl-3-bromoazetidine-3-carboxylate (15c)

Yellow oil, yield 25\%, $\mathrm{R}_{f}=0.16$ (petroleum ether/ $\mathrm{Et}_{2} \mathrm{O}$ 7/3). ${ }^{1} \mathrm{H} \mathrm{NMR}(300 \mathrm{MHz}$, $\left.\mathrm{CDCl}_{3}\right): \delta 0.96\left(9 \mathrm{H}, \mathrm{s}, \mathrm{C}\left(\mathrm{CH}_{3}\right)_{3}\right), 3.60-3.64(2 \mathrm{H}, \mathrm{m}, \mathrm{CH}(\mathrm{H}) \mathrm{NCH}(\mathrm{H})), 3.83(3 \mathrm{H}, \mathrm{s}$, $\left.\mathrm{OCH}_{3}\right), 3.91-3.95(2 \mathrm{H}, \mathrm{m}, \mathrm{CH}(H) \mathrm{NCH}(H)) .{ }^{13} \mathrm{C} \mathrm{NMR}\left(75 \mathrm{MHz}, \mathrm{CDCl}_{3}\right): \delta 24.1$, 44.9, 52.4, 53.5, 59.7, 170.9. IR (neat, $\left.\mathrm{cm}^{-1}\right): v_{\mathrm{C}=\mathrm{O}}=1736$. MS (ES, pos. mode): $m / z(\%): 250 / 252\left(\mathrm{M}+\mathrm{H}^{+}, 100\right)$. Anal. Calcd for $\mathrm{C}_{9} \mathrm{H}_{16} \mathrm{BrNO}_{2}(\%): \mathrm{C}, 43.22 ; \mathrm{H}$, 6.45; N, 5.60. Found (\%): C, 42.83; H, 6.61; N, 5.41.

Methyl 1-tert-pentyl-2-(bromomethyl)aziridine-2-carboxylate (14d)

Light yellow oil, yield 54\%, $\mathrm{R}_{f}=0.48$ (petroleum ether/Et $2 \mathrm{O} 7 / 3$ ). ${ }^{1} \mathrm{H}$ NMR (300 $\left.\mathrm{MHz}, \mathrm{CDCl}_{3}\right): \delta 0.90\left(3 \mathrm{H}, \mathrm{t}, J=7.6 \mathrm{~Hz}, \mathrm{CH}_{2} \mathrm{CH}_{3}\right), 0.94\left(3 \mathrm{H}, \mathrm{s}, \mathrm{CH}_{3} \mathrm{CCH}_{3}\right), 0.96$ $\left(3 \mathrm{H}, \mathrm{s}, \mathrm{CH}_{3} \mathrm{CCH}_{3}\right), 1.36-1.58\left(2 \mathrm{H}, \mathrm{m}, \mathrm{CH}_{2} \mathrm{CH}_{3}\right), 1.85(1 \mathrm{H}, \mathrm{s}, \mathrm{NCH}(\mathrm{H})), 2.59(1 \mathrm{H}$, s, $\mathrm{NCH}(H)), 3.05(1 \mathrm{H}, \mathrm{d}, J=9.9 \mathrm{~Hz}, \mathrm{BrCH}(\mathrm{H})), 3.77\left(3 \mathrm{H}, \mathrm{s}, \mathrm{OCH}_{3}\right), 4.07(1 \mathrm{H}, \mathrm{d}$, $J=10.2 \mathrm{~Hz}, \mathrm{BrCH}(H)) .{ }^{13} \mathrm{C} \mathrm{NMR}\left(75 \mathrm{MHz}, \mathrm{CDCl}_{3}\right): \delta 8.8,24.1,24.4,33.9,36.5$, 36.9, 44.3, 52.7, 57.4, 170.4. IR (neat, $\mathrm{cm}^{-1}$ ): $v_{\mathrm{C}=\mathrm{O}}=1732$. MS (ES, pos. mode): $m / z$ (\%): 264/266 (M+H', 100). Anal. Calcd for $\mathrm{C}_{10} \mathrm{H}_{18} \mathrm{BrNO}_{2}(\%): \mathrm{C}, 45.47 ; \mathrm{H}$, 6.87; N, 5.30. Found (\%): C, 45.38; H, 6.98; N, 5.02.

Methyl 1-tert-pentyl-3-bromoazetidine-3-carboxylate (15d)

Yellow oil, yield 26\%, $\mathrm{R}_{f}=0.31$ (petroleum ether/Et ${ }_{2} \mathrm{O} 7 / 3$ ). ${ }^{1} \mathrm{H} \mathrm{NMR}(300 \mathrm{MHz}$, $\left.\mathrm{CDCl}_{3}\right): \delta 0.83\left(3 \mathrm{H}, \mathrm{t}, J=7.4 \mathrm{~Hz}, \mathrm{CH}_{2} \mathrm{CH}_{3}\right), 0.88\left(6 \mathrm{H}, \mathrm{s}, \mathrm{C}\left(\mathrm{CH}_{3}\right)_{2}\right), 1.24(2 \mathrm{H}, \mathrm{q}, J$ 
$\left.=7.4 \mathrm{~Hz}, \mathrm{CH}_{2} \mathrm{CH}_{3}\right), 3.59-3.62(2 \mathrm{H}, \mathrm{m}, \mathrm{CH}(\mathrm{H}) \mathrm{NCH}(\mathrm{H})), 3.83\left(3 \mathrm{H}, \mathrm{s}, \mathrm{OCH}_{3}\right)$, 3.89-3.92 (2H, m, $\mathrm{CH}(H) \mathrm{NCH}(H)) .{ }^{13} \mathrm{C} \mathrm{NMR}\left(75 \mathrm{MHz}, \mathrm{CDCl}_{3}\right): \delta 8.6,20.1,31.6$, 45.3, 53.4, 54.8, 59.5, 171.0. IR (neat, $\mathrm{cm}^{-1}$ ): $v_{\mathrm{C}=\mathrm{O}}=1742$. MS (ES, pos. mode): $m / z(\%): 264 / 266\left(\mathrm{M}+\mathrm{H}^{+}, 100\right)$. Anal. Calcd for $\mathrm{C}_{10} \mathrm{H}_{18} \mathrm{BrNO}_{2}(\%): \mathrm{C}, 45.47 ; \mathrm{H}$, 6.87; N, 5.30. Found (\%): C, 45.12; H, 7.08; N, 5.42.

Isomerisation of alkyl 2-(bromomethyl)aziridines 14a-d to alkyl 3bromoazetidines 15a-d

As a representative example, the synthesis of methyl 1-tert-pentyl-3bromoazetidine-3-carboxylate (15d) is described here. Methyl 1-tert-pentyl-2(bromomethyl)aziridine-2-carboxylate (14d) (100 $\mathrm{mg}, 0.38 \mathrm{mmol})$ was dissolved in DMSO $(2 \mathrm{~mL})$ and heated at $70{ }^{\circ} \mathrm{C}$ for $5 \mathrm{~h}$. After cooling, the reaction mixture was poured into water $(2 \mathrm{~mL})$ and extracted with EtOAc $(3 \times 15 \mathrm{~mL})$. The combined organic extracts were washed with brine $(2 \times 15 \mathrm{~mL})$ and water $(15$ $\mathrm{mL})$. Drying $\left(\mathrm{MgSO}_{4}\right)$, filtration, evaporation of the solvent under reduced pressure and purification by preparative TLC on silica gel (hexane/EtOAc 9/1) afforded azetidine $\mathbf{1 5 d}$ in $50 \%$ yield.

Synthesis of alkyl 2-(cyanomethyl)aziridine-2-carboxylates 16a-c

As a representative example, the synthesis of methyl 1-tert-butyl-2(cyanomethyl)aziridine-2-carboxylate (16c) is described here. A solution of methyl 1-tert-butyl-2-(bromomethyl)aziridine-2-carboxylate (14c) (118 mg, 0.47 $\mathrm{mmol}$ ) and potassium cyanide (31 $\mathrm{mg}, 0.47 \mathrm{mmol})$ in DMSO $(2 \mathrm{~mL})$ was heated at $60{ }^{\circ} \mathrm{C}$ for $4 \mathrm{~h}$. After cooling, the reaction mixture was poured into water $(15$ $\mathrm{mL})$ and extracted with $\mathrm{Et}_{2} \mathrm{O}(3 \times 15 \mathrm{~mL})$. The combined organic extracts were washed with brine $(2 \times 15 \mathrm{~mL})$ and water $(15 \mathrm{~mL})$. Drying $\left(\mathrm{MgSO}_{4}\right)$, filtration, evaporation of the solvent under reduced pressure and purification by preparative TLC on silica gel (petroleum ether/EtOAc 8/2) afforded 2-(cyanomethyl)aziridine 16c in $57 \%$ yield.

Ethyl 1-tert-butyl-2-(cyanomethyl)aziridine-2-carboxylate (16a) 
Pink oil, yield 47\%, $\mathrm{R}_{f}=0.26$ (petroleum ether/Et $2 \mathrm{O} 7 / 3$ ). ${ }^{1} \mathrm{H}$ NMR (300 MHz, $\left.\mathrm{CDCl}_{3}\right): \delta 1.12\left(9 \mathrm{H}, \mathrm{s}, \mathrm{C}\left(\mathrm{CH}_{3}\right)_{3}\right), 1.34\left(3 \mathrm{H}, \mathrm{t}, J=7.2 \mathrm{~Hz}, \mathrm{CH}_{2} \mathrm{CH}_{3}\right), 1.95(1 \mathrm{H}, \mathrm{d}, J$ $=1.4 \mathrm{~Hz}, \mathrm{NCH}(\mathrm{H})), 2.60(1 \mathrm{H}, \mathrm{d}, J=1.1 \mathrm{~Hz}, \mathrm{NCH}(H)), 2.65(1 \mathrm{H}, \mathrm{d}, J=17.1 \mathrm{~Hz}$, $\mathrm{CH}(\mathrm{H}) \mathrm{CN}), 2.81(1 \mathrm{H}, \mathrm{d}, J=16.8 \mathrm{~Hz}, \mathrm{CH}(H) \mathrm{CN}), 4.21(1 \mathrm{H}, \mathrm{dq}, J=11.0 \mathrm{~Hz}, 7.2$ $\left.\mathrm{Hz}, \mathrm{CH}(\mathrm{H}) \mathrm{CH}_{3}\right), 4.87\left(1 \mathrm{H}, \mathrm{dq}, J=10.9 \mathrm{~Hz}, 7.2 \mathrm{~Hz}, \mathrm{CH}(H) \mathrm{CH}_{3}\right) .{ }^{13} \mathrm{C}$ NMR $(75$ $\left.\mathrm{MHz}, \mathrm{CDCl}_{3}\right): \delta 14.0,25.4,28.4,33.6,39.9,54.9,62.2,117.3,169.7$. IR (neat, $\left.\mathrm{cm}^{-1}\right): v_{\mathrm{CN}}=2253($ weak $), v_{\mathrm{C}=\mathrm{O}}=1733$. MS (ES, pos. mode): $\mathrm{m} / \mathrm{z}(\%): 211$ $\left(\mathrm{M}+\mathrm{H}^{+}, 100\right)$. Anal. Calcd for $\mathrm{C}_{11} \mathrm{H}_{18} \mathrm{~N}_{2} \mathrm{O}_{2}(\%)$ : C, 62.83; H, 8.63; N, 13.32 . Found (\%): C, 62.95; H, 8.78; N, 13.17.

Ethyl 1-tert-pentyl-2-(cyanomethyl)aziridine-2-carboxylate (16b)

Pink oil, yield 54\%, $\mathrm{R}_{f}=0.29$ (petroleum ether/Et $\mathrm{E}_{2} \mathrm{O} 7 / 3$ ). ${ }^{1} \mathrm{H}$ NMR (300 MHz, $\left.\left.\mathrm{CDCl}_{3}\right): \delta 0.92\left(3 \mathrm{H}, \mathrm{t}, J=7.4 \mathrm{~Hz}, \mathrm{CCH}_{2} \mathrm{CH}_{3}\right)\right), 1.00\left(3 \mathrm{H}, \mathrm{s}, \mathrm{CCH}_{3}\right), 1.01(3 \mathrm{H}, \mathrm{s}$, $\left.\left.\mathrm{CCH}_{3}\right), 1.33\left(3 \mathrm{H}, \mathrm{t}, J=7.2 \mathrm{~Hz}, \mathrm{OCH}_{2} \mathrm{CH}_{3}\right), 1.39-1.56\left(2 \mathrm{H}, \mathrm{m}, \mathrm{CCH}_{2} \mathrm{CH}_{3}\right)\right), 1.97$ $(1 \mathrm{H}, \mathrm{d}, J=1.7 \mathrm{~Hz}, \mathrm{NCH}(\mathrm{H})), 2.63(1 \mathrm{H}, \mathrm{d}, J=1.4 \mathrm{~Hz}, \mathrm{NCH}(H)), 2.66(1 \mathrm{H}, \mathrm{d}, J=$ $16.8 \mathrm{~Hz}, \mathrm{CH}(\mathrm{H}) \mathrm{CN}), 2.84(1 \mathrm{H}, \mathrm{d}, J=16.8 \mathrm{~Hz}, \mathrm{CH}(H) \mathrm{CN}), 4.21(1 \mathrm{H}, \mathrm{dq}, J=10.7$ $\left.\mathrm{Hz}, 7.2 \mathrm{~Hz}, \mathrm{OCH}(\mathrm{H}) \mathrm{CH}_{3}\right), 4.26\left(1 \mathrm{H}, \mathrm{dq}, J=10.7 \mathrm{~Hz}, 7.2 \mathrm{~Hz}, \mathrm{OCH}(H) \mathrm{CH}_{3}\right) .{ }^{13} \mathrm{C}$ NMR (75 MHz, $\left.\mathrm{CDCl}_{3}\right): \delta 8.8,14.0,23.9,24.6,25.3,33.8,37.0,39.2,57.2,62.2$, 117.3, 169.9. IR (neat, $\mathrm{cm}^{-1}$ ): $v_{\mathrm{CN}}=2253$ (weak), $v_{\mathrm{C}=\mathrm{O}}=1734$. MS (ES, pos. mode): $m / z(\%): 225\left(\mathrm{M}+\mathrm{H}^{+}, 100\right)$. Anal. Calcd for $\mathrm{C}_{12} \mathrm{H}_{20} \mathrm{~N}_{2} \mathrm{O}_{2}(\%): \mathrm{C}, 64.26 ; \mathrm{H}$, 8.99; N, 12.49. Found (\%): C, 64.01; H, 8.84; N, 12.61.

Methyl 1-tert-butyl-2-(cyanomethyl)aziridine-2-carboxylate (16c)

Yellow oil, yield 57\%, $\mathrm{R}_{f}=0.24$ (petroleum ether/EtOAc 7/3). ${ }^{1} \mathrm{H}$ NMR (300 $\left.\mathrm{MHz}_{\mathrm{CDCl}}\right): \delta 1.11\left(9 \mathrm{H}, \mathrm{s},\left(\mathrm{CH}_{3}\right)_{3}\right), 1.96(1 \mathrm{H}, \mathrm{d}, J=1.4 \mathrm{~Hz}, \mathrm{NCH}(\mathrm{H})), 2.60$ $(1 \mathrm{H}, \mathrm{d}, J=1.4 \mathrm{~Hz}, \mathrm{NCH}(H)), 2.67(1 \mathrm{H}, \mathrm{d}, J=16.8 \mathrm{~Hz}, \mathrm{CH}(\mathrm{H}) \mathrm{CN}), 2.81(1 \mathrm{H}, \mathrm{d}, J$ $=17.1 \mathrm{~Hz}, \mathrm{CH}(H) \mathrm{CN}), 3.79\left(3 \mathrm{H}, \mathrm{s}, \mathrm{OCH}_{3}\right) .{ }^{13} \mathrm{C} \mathrm{NMR}\left(75 \mathrm{MHz}, \mathrm{CDCl}_{3}\right): \delta 25.2$, $28.4,33.7,39.7,53.0,54.8,117.2,170.2$. IR (neat, $\mathrm{cm}^{-1}$ ): $v_{\mathrm{CN}}=2253$ (weak), $v_{\mathrm{C}=\mathrm{O}}$ $=1728$. MS (ES, pos. mode): $m / z(\%): 197\left(\mathrm{M}+\mathrm{H}^{+}, 100\right)$. Anal. Calcd for $\mathrm{C}_{10} \mathrm{H}_{16} \mathrm{~N}_{2} \mathrm{O}_{2}$ (\%): C, 61.20; H, 8.22; N, 14.27. Found (\%): C, 61.14; H, 8.43; N, 14.25 . 
Synthesis of alkyl 2-(thiocyanomethyl)aziridine-2-carboxylates $\mathbf{1 7 a - b}$

As a representative example, the synthesis of ethyl 1-tert-butyl-2(thiocyanomethyl)aziridine-2-carboxylate (17a) is described here. A solution of ethyl 1-tert-butyl-2-(bromomethyl)aziridine-2-carboxylate (14a) (132 mg, 0.5 $\mathrm{mmol})$ and potassium thiocyanate $(97 \mathrm{mg}, 1 \mathrm{mmol})$ in DMF $(2 \mathrm{~mL})$ was heated at $70{ }^{\circ} \mathrm{C}$ for $2 \mathrm{~h}$. After cooling, the reaction mixture was poured into water (15 mL) and extracted with EtOAc $(3 \times 15 \mathrm{~mL})$. The combined organic extracts were washed with brine $(2 \times 15 \mathrm{~mL})$ and water $(15 \mathrm{~mL})$. Drying $\left(\mathrm{MgSO}_{4}\right)$, filtration, evaporation of the solvent under reduced pressure and purification by preparative TLC on silica gel (petroleum ether/Et $\mathrm{E}_{2} \mathrm{O}$ 7/3) afforded 2(thiocyanomethyl)aziridine $\mathbf{1 7 a}$ in $48 \%$ yield.

Ethyl 1-tert-butyl-2-(thiocyanomethyl)aziridine-2-carboxylate (17a)

Yellow oil, yield 48\%, $\mathrm{R}_{f}=0.31$ (petroleum ether/Et ${ }_{2} \mathrm{O}$ 7/3). ${ }^{1} \mathrm{H} \mathrm{NMR}(300 \mathrm{MHz}$, $\left.\mathrm{CDCl}_{3}\right): \delta 1.10\left(9 \mathrm{H}, \mathrm{s},\left(\mathrm{CH}_{3}\right)_{3}\right), 1.33\left(3 \mathrm{H}, \mathrm{t}, J=7.2 \mathrm{~Hz}, \mathrm{CH}_{2} \mathrm{CH}_{3}\right), 2.05(1 \mathrm{H}, \mathrm{d}, J=$ $1.2 \mathrm{~Hz}, \mathrm{NCH}(H)), 2.64(1 \mathrm{H}, \mathrm{s}, \mathrm{NCH}(\mathrm{H})), 3.28(1 \mathrm{H}, \mathrm{d}, J=12.9 \mathrm{~Hz}, \mathrm{CH}(\mathrm{H}) \mathrm{SCN})$, $3.39(1 \mathrm{H}, \mathrm{d}, J=12.9 \mathrm{~Hz}, \mathrm{CH}(H) \mathrm{SCN}), 4.20(1 \mathrm{H}, \mathrm{dq}, J=11.0 \mathrm{~Hz}, 7.2 \mathrm{~Hz}$, $\left.\mathrm{CH}(\mathrm{H}) \mathrm{CH}_{3}\right), 4.28\left(1 \mathrm{H}, \mathrm{dq}, J=10.7 \mathrm{~Hz}, 7.2 \mathrm{~Hz}, \mathrm{CH}(H) \mathrm{CH}_{3}\right) .{ }^{13} \mathrm{C} \mathrm{NMR}(75 \mathrm{MHz}$, $\mathrm{CDCl}_{3}$ ): $\delta 14.0,28.3,33.4,40.2,42.7,54.9,62.2,113.1,169.7$. IR (neat, $\mathrm{cm}^{-1}$ ): $v_{\mathrm{SCN}}=2154, v_{\mathrm{C}=\mathrm{O}}=1735$. MS (ES, pos. mode): $m / z(\%): 243\left(\mathrm{M}+\mathrm{H}^{+}, 100\right)$. Anal. Calcd for $\mathrm{C}_{11} \mathrm{H}_{18} \mathrm{~N}_{2} \mathrm{O}_{2} \mathrm{~S}(\%)$ : C, 54.52; H, 7.49; N, 11.56. Found (\%): C, 54.69; H, $7.61 ; \mathrm{N}, 11.37$.

Ethyl 1-tert-pentyl-2-(thiocyanomethyl)aziridine-2-carboxylate $(\mathbf{1 7 b})$

Yellow oil, yield 44\%, $\mathrm{R}_{f}=0.30$ (petroleum ether/EtOAc 9/1). ${ }^{1} \mathrm{H}$ NMR (300 $\left.\mathrm{MHz}, \mathrm{CDCl}_{3}\right): \delta 0.91\left(3 \mathrm{H}, \mathrm{t}, J=7.7 \mathrm{~Hz}, \mathrm{CCH}_{2} \mathrm{CH}_{3}\right), 0.98\left(3 \mathrm{H}, \mathrm{s}, \mathrm{CCH}_{3}\right), 1.01$ $\left(3 \mathrm{H}, \mathrm{s}, \mathrm{CCH}_{3}\right), 1.33\left(3 \mathrm{H}, \mathrm{t}, J=7.2 \mathrm{~Hz}, \mathrm{OCH}_{2} \mathrm{CH}_{3}\right), 1.37-1.58\left(2 \mathrm{H}, \mathrm{m}, \mathrm{CCH}_{2} \mathrm{CH}_{3}\right)$, $2.07(1 \mathrm{H}, \mathrm{s}, \mathrm{NCH}(H)), 2.66(1 \mathrm{H}, \mathrm{s}, \mathrm{NCH}(H)), 3.32(1 \mathrm{H}, \mathrm{d}, J=12.9 \mathrm{~Hz}$, $\mathrm{CH}(\mathrm{H}) \mathrm{SCN}), 3.43(1 \mathrm{H}, \mathrm{d}, J=13.2 \mathrm{~Hz}, \mathrm{CH}(H) \mathrm{SCN}), 4.19(1 \mathrm{H}, \mathrm{dq}, J=10.7 \mathrm{~Hz}$, $\left.7.2 \mathrm{~Hz}, \mathrm{OCH}(\mathrm{H}) \mathrm{CH}_{3}\right), 4.27\left(1 \mathrm{H}, \mathrm{dq}, J=11.0 \mathrm{~Hz}, 7.2 \mathrm{~Hz}, \mathrm{OCH}(H) \mathrm{CH}_{3}\right) .{ }^{13} \mathrm{C} \mathrm{NMR}$ $\left(75 \mathrm{MHz}, \mathrm{CDCl}_{3}\right): \delta 8.8,14.0,23.9,24.7,33.4,36.8,40.3,42.0,57.3,62.2,113.1$, 
169.9. IR (neat, $\left.\mathrm{cm}^{-1}\right): v_{\mathrm{SCN}}=2155, v_{\mathrm{C}=\mathrm{O}}=1735$. MS (ES, pos. mode): $\mathrm{m} / z(\%)$ : $257\left(\mathrm{M}+\mathrm{H}^{+}\right.$, 100). Anal. Calcd for $\mathrm{C}_{12} \mathrm{H}_{20} \mathrm{~N}_{2} \mathrm{O}_{2} \mathrm{~S}(\%)$ : C, 56.22; H, 7.86; N, 10.93 . Found (\%): C, 55.98; H, 8.02; N, 10.68.

Synthesis of alkyl 2-(azidomethyl)aziridine-2-carboxylates 18a-d

As a representative example, the synthesis of methyl 1-tert-butyl-2(azidomethyl)aziridine-2-carboxylate (18c) is described here. Methyl 1-tertbutyl-2-(bromomethyl)aziridine-2-carboxylate (14c) (250 $\mathrm{mg}, 1 \mathrm{mmol})$ and sodium azide (130 mg, $2 \mathrm{mmol})$ were heated in DMSO $(5 \mathrm{~mL})$ at $60{ }^{\circ} \mathrm{C}$ for $3 \mathrm{~h}$. After cooling, the reaction mixture was poured into water $(15 \mathrm{~mL})$ and extracted with $\mathrm{Et}_{2} \mathrm{O}(3 \times 25 \mathrm{~mL})$. The combined organic extracts were washed with brine $(2 \times 15 \mathrm{~mL})$ and water $(15 \mathrm{~mL})$. Drying with $\mathrm{MgSO}_{4}$, filtration of the drying agent, evaporation of the solvent under reduced pressure and purification by flash chromatography on silica gel (Hexane/Et ${ }_{2} \mathrm{O} 9 / 1$ - 7/3) afforded 2(azidomethyl)aziridine $\mathbf{1 8 c}$ in $91 \%$ yield.

Ethyl 1-tert-butyl-2-(azidomethyl)aziridine-2-carboxylate (18a)

Yellow oil, yield 58\%, $\mathrm{R}_{f}=0.47$ (petroleum ether/EtOAc 8/2). ${ }^{1} \mathrm{H}$ NMR (300 $\left.\mathrm{MHz}, \mathrm{CDCl}_{3}\right): \delta 1.09\left(9 \mathrm{H}, \mathrm{s}, \mathrm{C}\left(\mathrm{CH}_{3}\right)_{3}\right), 1.33\left(3 \mathrm{H}, \mathrm{t}, J=7.2 \mathrm{~Hz}, \mathrm{CH}_{2} \mathrm{CH}_{3}\right), 1.88$ $(1 \mathrm{H}, \mathrm{d}, J=1.7 \mathrm{~Hz}, \mathrm{NCH}(\mathrm{H})), 2.47-2.48(1 \mathrm{H}, \mathrm{m}, \mathrm{NCH}(H)), 3.26(1 \mathrm{H}, \mathrm{d}, J=12.7$ $\left.\mathrm{Hz}, \mathrm{CH}(\mathrm{H}) \mathrm{N}_{3}\right), 3.68\left(1 \mathrm{H}, \mathrm{d}, J=12.7 \mathrm{~Hz}, \mathrm{CH}(H) \mathrm{N}_{3}\right), 4.19(1 \mathrm{H}, \mathrm{dq}, J=10.7 \mathrm{~Hz}$, $\left.7.2 \mathrm{~Hz}, \mathrm{OCH}(\mathrm{H}) \mathrm{CH}_{3}\right), 4.26\left(1 \mathrm{H}, \mathrm{dq}, J=10.7 \mathrm{~Hz}, 7.2 \mathrm{~Hz}, \mathrm{OCH}(H) \mathrm{CH}_{3}\right) .{ }^{13} \mathrm{C} \mathrm{NMR}$ (75 MHz, $\mathrm{CDCl}_{3}$ ): $\delta 14.0,28.3,32.0,43.1,54.6,55.8,61.8,170.4$. IR (neat, $\mathrm{cm}^{-1}$ ): $v_{\mathrm{N} 3}=2101, v_{\mathrm{C}=\mathrm{O}}=1734$. MS (ES, pos. mode): $m / z(\%): 227\left(\mathrm{M}+\mathrm{H}^{+}, 100\right)$. Anal. Calcd for $\mathrm{C}_{10} \mathrm{H}_{18} \mathrm{~N}_{4} \mathrm{O}_{2}(\%)$ : C, 53.08; H, 8.02; N, 24.76. Found (\%): C, 52.77; H, $8.17 ; \mathrm{N}, 24.45$.

Ethyl 1-tert-pentyl-2-(azidomethyl)aziridine-2-carboxylate (18b)

Yellow oil, yield 93\%, $\mathrm{R}_{f}=0.50$ (petroleum ether/EtOAc 9/1). ${ }^{1} \mathrm{H}$ NMR (300 $\left.\mathrm{MHz}, \mathrm{CDCl}_{3}\right): \delta 0.91\left(3 \mathrm{H}, \mathrm{t}, J=7.4 \mathrm{~Hz}, \mathrm{CCH}_{2} \mathrm{CH}_{3}\right), 0.97\left(3 \mathrm{H}, \mathrm{s}, \mathrm{CCH}_{3}\right), 0.99$ $\left(3 \mathrm{H}, \mathrm{s}, \mathrm{CCH}_{3}\right), 1.32\left(3 \mathrm{H}, \mathrm{t}, J=7.2 \mathrm{~Hz}, \mathrm{OCH}_{2} \mathrm{CH}_{3}\right), 1.46(1 \mathrm{H}, \mathrm{dq}, J=13.8 \mathrm{~Hz}, 7.3$ 
$\left.\mathrm{Hz}, \mathrm{CCH}(\mathrm{H}) \mathrm{CH}_{3}\right), 1.51\left(1 \mathrm{H}, \mathrm{dq}, J=13.6 \mathrm{~Hz}, 7.5 \mathrm{~Hz}, \mathrm{CCH}(H) \mathrm{CH}_{3}\right), 1.90(1 \mathrm{H}, \mathrm{d}, J$ $=1.1 \mathrm{~Hz}, \mathrm{NCH}(\mathrm{H})), 2.50-2.51(1 \mathrm{H}, \mathrm{m}, \mathrm{NCH}(H)), 3.26(1 \mathrm{H}, \mathrm{d}, J=12.7 \mathrm{~Hz}$, $\left.\mathrm{C} H(\mathrm{H}) \mathrm{N}_{3}\right), 3.70\left(1 \mathrm{H}, \mathrm{d}, J=12.7 \mathrm{~Hz}, \mathrm{CH}(H) \mathrm{N}_{3}\right), 4.19(1 \mathrm{H}, \mathrm{dq}, J=11.0 \mathrm{~Hz}, 7.2$ $\left.\mathrm{Hz}, \mathrm{OCH}(\mathrm{H}) \mathrm{CH}_{3}\right), 4.25\left(1 \mathrm{H}, \mathrm{dq}, J=10.7 \mathrm{~Hz}, 7.2 \mathrm{~Hz}, \mathrm{OCH}(H) \mathrm{CH}_{3}\right) .{ }^{13} \mathrm{C} \mathrm{NMR}$ $\left(75 \mathrm{MHz} \mathrm{CDCl}_{3}\right): \delta 8.8,14.0,24.0,24.6,32.1,36.6,42.5,55.7,57.0,61.7,170.7$. IR (neat, $\left.\mathrm{cm}^{-1}\right): v_{\mathrm{N} 3}=2101, v_{\mathrm{C}=\mathrm{O}}=1734$. MS (ES, pos. mode): $\mathrm{m} / \mathrm{z}(\%): 241$ $\left(\mathrm{M}+\mathrm{H}^{+}\right.$, 100). Anal. Calcd for $\mathrm{C}_{11} \mathrm{H}_{20} \mathrm{~N}_{4} \mathrm{O}_{2}(\%)$ : C, 54.98; H, 8.39; N, 23.32. Found (\%): C, 54.61; H, 8.53; N, 23.14.

Methyl 1-tert-butyl-2-(azidomethyl)aziridine-2-carboxylate (18c)

Light yellow oil, yield 91\%, $\mathrm{R}_{f}=0.53$ (hexane/Et ${ }_{2} \mathrm{O} 7 / 3$ ). ${ }^{1} \mathrm{H}$ NMR $(300 \mathrm{MHz}$, $\left.\mathrm{CDCl}_{3}\right): \delta 1.09\left(9 \mathrm{H}, \mathrm{s}, \mathrm{C}\left(\mathrm{CH}_{3}\right)_{3}\right), 1.90(1 \mathrm{H}, \mathrm{d}, J=1.1 \mathrm{~Hz}, \mathrm{NCH}(\mathrm{H})), 2.47(1 \mathrm{H}, \mathrm{s}$, $\mathrm{NCH}(H)), 3.29\left(1 \mathrm{H}, \mathrm{d}, J=12.7 \mathrm{~Hz}, \mathrm{CH}(\mathrm{H}) \mathrm{N}_{3}\right), 3.67(1 \mathrm{H}, \mathrm{d}, J=12.7 \mathrm{~Hz}$, $\left.\mathrm{CH}(H) \mathrm{N}_{3}\right), 3.77\left(3 \mathrm{H}, \mathrm{s}, \mathrm{OCH}_{3}\right) .{ }^{13} \mathrm{C} \mathrm{NMR}\left(75 \mathrm{MHz}, \mathrm{CDCl}_{3}\right): \delta 28.2,32.0,42.9$, 52.7, 54.6, 55.6, 61.8, 171.0. IR (neat, $\left.\mathrm{cm}^{-1}\right): v_{\mathrm{N} 3}=2105, v_{\mathrm{C}=\mathrm{O}}=1740$. MS (ES, pos. mode): $m / z(\%): 213\left(\mathrm{M}+\mathrm{H}^{+}, 100\right)$. Anal. Calcd for $\mathrm{C}_{9} \mathrm{H}_{16} \mathrm{~N}_{4} \mathrm{O}_{2}(\%)$ : C, 50.93; H, 7.60; N, 26.40. Found (\%): C, 50.64; H, 7.47; N, 26.51.

Methyl 1-tert-pentyl-2-(azidomethyl)aziridine-2-carboxylate (18d)

Yellow oil, yield 64\%, $\mathrm{R}_{f}=0.25$ (petroleum ether/Et $\mathrm{E}_{2} \mathrm{O}$ 9/1). ${ }^{1} \mathrm{H}$ NMR (300 MHz, $\left.\mathrm{CDCl}_{3}\right): \delta 0.91\left(3 \mathrm{H}, \mathrm{t}, J=7.6 \mathrm{~Hz}, \mathrm{CCH}_{2} \mathrm{CH}_{3}\right), 0.96\left(3 \mathrm{H}, \mathrm{s}, \mathrm{CCH}_{3}\right), 0.98(3 \mathrm{H}, \mathrm{s}$, $\left.\mathrm{CCH}_{3}\right), 1.45\left(1 \mathrm{H}, \mathrm{dq}, J=13.6 \mathrm{~Hz}, 7.3 \mathrm{~Hz}, \mathrm{CCH}(\mathrm{H}) \mathrm{CH}_{3}\right), 1.50(1 \mathrm{H}, \mathrm{dq}, J=13.6$ $\left.\mathrm{Hz}, 7.7 \mathrm{~Hz}, \mathrm{CCH}(H) \mathrm{CH}_{3}\right), 1.92(1 \mathrm{H}, \mathrm{d}, J=1.4 \mathrm{~Hz}, \mathrm{NCH}(\mathrm{H})), 2.50-2.51(1 \mathrm{H}, \mathrm{m}$, $\mathrm{NCH}(H)), 3.28\left(1 \mathrm{H}, \mathrm{d}, J=12.7 \mathrm{~Hz}, \mathrm{CH}(\mathrm{H}) \mathrm{N}_{3}\right), 3.69(1 \mathrm{H}, \mathrm{d}, J=12.7 \mathrm{~Hz}$, $\left.\mathrm{CH}(H) \mathrm{N}_{3}\right), 3.77\left(3 \mathrm{H}, \mathrm{s}, \mathrm{OCH}_{3}\right) .{ }^{13} \mathrm{C} \mathrm{NMR}\left(75 \mathrm{MHz}, \mathrm{CDCl}_{3}\right): \delta 8.8,23.9,24.5$, 32.1, 36.6, 42.2, 52.6, 55.6, 56.9, 171.3. IR (neat, $\left.\mathrm{cm}^{-1}\right): v_{\mathrm{N} 3}=2100, v_{\mathrm{C}=\mathrm{O}}=1732$. MS (ES, pos. mode): $m / z(\%): 227\left(\mathrm{M}+\mathrm{H}^{+}, 100\right)$. Anal. Calcd for $\mathrm{C}_{10} \mathrm{H}_{18} \mathrm{~N}_{4} \mathrm{O}_{2}$ (\%): C, 53.08; H, 8.02; N, 24.76. Found (\%): C, 52.85; H, 8.24; N, 24.59.

Synthesis of alkyl 2-(phenoxymethyl)aziridine-2-carboxylates 19a-b 
As a representative example, the synthesis of ethyl 1-tert-butyl-2(phenoxymethyl)aziridine-2-carboxylate (19a) is described here. Ethyl 1-tertbutyl-2-(bromomethyl)aziridine-2-carboxylate (14a) (80 $\mathrm{mg}, 0.3 \mathrm{mmol}$ ) was added to a mixture of phenol (57 mg, $0.6 \mathrm{mmol})$ and $\mathrm{K}_{2} \mathrm{CO}_{3}(168 \mathrm{mg}, 1.2 \mathrm{mmol})$ in DMSO (4 mL) and the reaction mixture was heated at $60{ }^{\circ} \mathrm{C}$ for $8 \mathrm{~h}$. After cooling, the reaction mixture was poured into water $(15 \mathrm{~mL})$ and extracted with $\mathrm{Et}_{2} \mathrm{O}(3 \times 15 \mathrm{~mL})$. The combined organic extracts were washed with brine $(2 \times 15$ $\mathrm{mL}$ ), saturated $\mathrm{NaOH}$ (aq.) solution $(2 \times 10 \mathrm{~mL})$ and water $(15 \mathrm{~mL})$. Drying $\left(\mathrm{MgSO}_{4}\right)$, filtration, evaporation of the solvent under reduced pressure and purification by preparative TLC on silica gel (petroleum ether/EtOAc 9/1) afforded 2-(phenoxymethyl)aziridine 19a in 60\% yield.

Ethyl 1-tert-butyl-2-(phenoxymethyl)aziridine-2-carboxylate (19a)

Yellow oil, yield $60 \%, \mathrm{R}_{f}=0.33$ (petroleum ether /EtOAc 9/1). ${ }^{1} \mathrm{H}$ NMR (300 $\left.\mathrm{MHz}, \mathrm{CDCl}_{3}\right): \delta 1.11\left(9 \mathrm{H}, \mathrm{s},\left(\mathrm{CH}_{3}\right)_{3}\right), 1.25\left(3 \mathrm{H}, \mathrm{t}, J=7.2 \mathrm{~Hz}, \mathrm{CH}_{2} \mathrm{CH}_{3}\right), 1.92(1 \mathrm{H}$, $\mathrm{d}, J=1.1 \mathrm{~Hz}, \mathrm{NCH}(\mathrm{H})), 2.49(1 \mathrm{H}, \mathrm{s}, \mathrm{NCH}(H)), 3.72(1 \mathrm{H}, \mathrm{d}, J=9.4 \mathrm{~Hz}$, $\mathrm{CCH}(\mathrm{H}) \mathrm{O}), 4.18\left(1 \mathrm{H}, \mathrm{dq}, J=10.7 \mathrm{~Hz}, 7.2 \mathrm{~Hz}, \mathrm{OCH}(\mathrm{H}) \mathrm{CH}_{3}\right), 4.22(1 \mathrm{H}, \mathrm{dq}, J=$ $\left.10.7 \mathrm{~Hz}, 7.2 \mathrm{~Hz}, \mathrm{OCH}(H) \mathrm{CH}_{3}\right), 4.60(1 \mathrm{H}, \mathrm{d}, J=9.6 \mathrm{~Hz}, \mathrm{CCH}(H) \mathrm{O}), 6.86-6.97$ $\left(3 \mathrm{H}, \mathrm{m}, 3 \times \mathrm{CH}_{\text {arom }}\right), 7.23-7.30\left(2 \mathrm{H}, \mathrm{m}, 2 \times \mathrm{CH}_{\text {arom }}\right) .{ }^{13} \mathrm{C} \mathrm{NMR}\left(75 \mathrm{MHz}, \mathrm{CDCl}_{3}\right): \delta$ 14.1, 28.2, 31.7, 43.6, 54.6, 61.5, 72.1, 114.9, 121.1, 129.5, 158.7, 170.4. IR (neat, $\left.\mathrm{cm}^{-1}\right): v_{\mathrm{C}=\mathrm{O}}=1730$. MS (ES, pos. mode): $\mathrm{m} / z(\%): 278\left(\mathrm{M}+\mathrm{H}^{+}, 100\right)$. Anal. Calcd for $\mathrm{C}_{16} \mathrm{H}_{23} \mathrm{NO}_{3}(\%)$ : C, 69.29; $\mathrm{H}, 8.36 ; \mathrm{N}, 5.05$. Found (\%): C, 69.43; H, 8.61; N, 4.76 .

Ethyl 1-tert-pentyl-2-(phenoxymethyl)aziridine-2-carboxylate (19b)

Yellow oil, yield 67\%, $\mathrm{R}_{f}=0.19$ (petroleum ether/EtOAc 9/1). ${ }^{1} \mathrm{H}$ NMR (300 $\left.\mathrm{MHz} \mathrm{CDCl}_{3}\right): \delta 0.94\left(3 \mathrm{H}, \mathrm{t}, J=7.4 \mathrm{~Hz}, \mathrm{CCH}_{2} \mathrm{CH}_{3}\right), 0.97\left(3 \mathrm{H}, \mathrm{s}, \mathrm{CCH}_{3}\right), 1.00$ $\left(3 \mathrm{H}, \mathrm{s}, \mathrm{CCH}_{3}\right), 1.25\left(3 \mathrm{H}, \mathrm{t}, J=7.2 \mathrm{~Hz}, \mathrm{OCH}_{2} \mathrm{CH}_{3}\right), 1.45-1.59\left(2 \mathrm{H}, \mathrm{m}, \mathrm{CCH}_{2} \mathrm{CH}_{3}\right)$, $1.93(1 \mathrm{H}, \mathrm{d}, J=1.4 \mathrm{~Hz}, \mathrm{NCH}(\mathrm{H})), 2.53(1 \mathrm{H}, \mathrm{s}, \mathrm{NCH}(H)), 3.70(1 \mathrm{H}, \mathrm{d}, J=9.4 \mathrm{~Hz}$, $\mathrm{CCH}(\mathrm{H}) \mathrm{O}), 4.18\left(1 \mathrm{H}, \mathrm{dq}, J=10.7 \mathrm{~Hz}, 7.2 \mathrm{~Hz}, \mathrm{OCH}(\mathrm{H}) \mathrm{CH}_{3}\right), 4.21(1 \mathrm{H}, \mathrm{dq}, J=$ $\left.11.0 \mathrm{~Hz}, 7.2 \mathrm{~Hz}, \mathrm{OCH}(H) \mathrm{CH}_{3}\right), 4.61(1 \mathrm{H}, \mathrm{dd}, J=9.4 \mathrm{~Hz}, 0.8 \mathrm{~Hz}, \mathrm{CCH}(H) \mathrm{O})$, 6.85-6.97 (3H, m, $\left.3 \times \mathrm{CH}_{\text {arom }}\right), 7.23-7.30\left(2 \mathrm{H}, \mathrm{m}, 2 \times \mathrm{CH}_{\text {arom }}\right) .{ }^{13} \mathrm{C} \mathrm{NMR}(75 \mathrm{MHz}$, 
$\left.\mathrm{CDCl}_{3}\right): \delta 8.8,14.1,24.0,24.5,31.9,36.4,42.8,56.9,61.4,72.1,114.9,121.1$, 129.5, 158.8, 170.7. IR (neat, $\left.\mathrm{cm}^{-1}\right): v_{\mathrm{C}=\mathrm{O}}=1730$. MS (ES, pos. mode): $m / z(\%)$ : $292\left(\mathrm{M}+\mathrm{H}^{+}, 100\right)$. Anal. Calcd for $\mathrm{C}_{17} \mathrm{H}_{25} \mathrm{NO}_{3}(\%)$ : C, 70.07; $\mathrm{H}, 8.65 ; \mathrm{N}, 4.81$. Found (\%): C, 69.91; H, 8.83; N, 4.54.

Synthesis of ethyl 1-tert-butyl-3-cyanoazetidine-3-carboxylate (21a)

A solution of ethyl 1-tert-butyl-3-bromoazetidine-3-carboxylate (15a) (132 mg, $0.5 \mathrm{mmol})$ and potassium cyanide $(65 \mathrm{mg}, 1 \mathrm{mmol})$ in DMSO $(2 \mathrm{~mL})$ was heated at $60{ }^{\circ} \mathrm{C}$ for $4 \mathrm{~h}$. After cooling, the reaction mixture was poured into water $(20$ $\mathrm{mL})$ and extracted with $\mathrm{Et}_{2} \mathrm{O}(3 \times 15 \mathrm{~mL})$. The combined organic extracts were washed with brine $(2 \times 15 \mathrm{~mL})$ and water $(15 \mathrm{~mL})$. Drying $\left(\mathrm{MgSO}_{4}\right)$, filtration, evaporation of the solvent under reduced pressure and purification by preparative TLC on silica gel (petroleum ether/EtOAc 8/2) afforded 3-cyanoazetidine 21a in $79 \%$ yield. Yellow oil, $\mathrm{R}_{f}=0.43$ (petroleum ether/EtOAc 7/3). ${ }^{1} \mathrm{H}$ NMR (300 $\left.\mathrm{MHz}, \mathrm{CDCl}_{3}\right): \delta 0.96\left(9 \mathrm{H}, \mathrm{s}, \mathrm{C}\left(\mathrm{CH}_{3}\right)_{3}\right), 1.34\left(3 \mathrm{H}, \mathrm{t}, J=7.2 \mathrm{~Hz}, \mathrm{CH}_{2} \mathrm{CH}_{3}\right), 3.62$ $(2 \mathrm{H}, \mathrm{d}, J=7.2 \mathrm{~Hz}, \mathrm{CH}(\mathrm{H}) \mathrm{NCH}(\mathrm{H})), 3.72(2 \mathrm{H}, \mathrm{d}, J=7.4 \mathrm{~Hz}, \mathrm{CH}(H) \mathrm{NCH}(H))$, $4.30\left(2 \mathrm{H}, \mathrm{q}, J=7.2 \mathrm{~Hz}, \mathrm{CH}_{2} \mathrm{CH}_{3}\right) .{ }^{13} \mathrm{C} \mathrm{NMR}\left(75 \mathrm{MHz}, \mathrm{CDCl}_{3}\right): \delta 14.0,23.8,34.6$, 52.2, 54.3, 63.2, 119.0, 167.0. IR (neat, $\mathrm{cm}^{-1}$ ): $v_{\mathrm{CN}}=2246$ (weak), $v_{\mathrm{C}=\mathrm{O}}=1742$. MS (ES, pos. mode): $m / z(\%): 211\left(\mathrm{M}+\mathrm{H}^{+}, 100\right)$. Anal. Calcd for $\mathrm{C}_{11} \mathrm{H}_{18} \mathrm{~N}_{2} \mathrm{O}_{2}$ (\%): C, 62.83; H, 8.63; N, 13.32. Found (\%): C, 62.91; H, 8.79; N, 13.01.

Synthesis of alkyl 3-thiocyanoazetidine-3-carboxylates 22a-b

As a representative example, the synthesis of ethyl 1-tert-butyl-3thiocyanoazetidine-3-carboxylate (22a) is described here. A solution of ethyl 1tert-butyl-3-bromoazetidine-3-carboxylate (15a) (94 $\mathrm{mg}, 0.36 \mathrm{mmol})$ and potassium thiocyanate $(52 \mathrm{mg}, 0.53 \mathrm{mmol})$ in DMSO $(2 \mathrm{~mL})$ was heated at $60{ }^{\circ} \mathrm{C}$ for $3 \mathrm{~h}$. After cooling, the reaction mixture was poured into water $(15 \mathrm{~mL})$ and extracted with $\mathrm{Et}_{2} \mathrm{O}(3 \times 20 \mathrm{~mL})$. The combined organic extracts were washed with brine $(2 \times 15 \mathrm{~mL})$ and water $(15 \mathrm{~mL})$. Drying $\left(\mathrm{MgSO}_{4}\right)$, filtration, evaporation of the solvent under reduced pressure and purification by preparative TLC on silica gel (petroleum ether/Et ${ }_{2} \mathrm{O} 7 / 3$ ) afforded 3-thiocyanoazetidine 22a in $59 \%$ yield. 
Ethyl 1-tert-butyl-3-thiocyanoazetidine-3-carboxylate (22a)

Yellow oil, yield 59\%, $\mathrm{R}_{f}=0.17$ (petroleum ether/Et ${ }_{2} \mathrm{O} 7 / 3$ ). ${ }^{1} \mathrm{H}$ NMR (300 MHz, $\left.\mathrm{CDCl}_{3}\right): \delta 0.98\left(9 \mathrm{H}, \mathrm{s}, \mathrm{C}\left(\mathrm{CH}_{3}\right)_{3}\right), 1.35\left(3 \mathrm{H}, \mathrm{t}, J=7.2 \mathrm{~Hz}, \mathrm{OCH}_{2} \mathrm{CH}_{3}\right), 3.44(2 \mathrm{H}, \mathrm{d}$, $J=8.8 \mathrm{~Hz}, \mathrm{CH}(\mathrm{H}) \mathrm{NCH}(\mathrm{H})), 3.83(2 \mathrm{H}, \mathrm{d}, J=8.8 \mathrm{~Hz}, \mathrm{CH}(H) \mathrm{NCH}(H)), 4.32(2 \mathrm{H}$, q, $\left.J=7.2 \mathrm{~Hz}, \mathrm{OCH}_{2} \mathrm{CH}_{3}\right) .{ }^{13} \mathrm{C} \mathrm{NMR}\left(75 \mathrm{MHz}, \mathrm{CDCl}_{3}\right): \delta 14.1,24.0,47.4,52.4$, 55.7, 63.0, 109.9, 169.2. IR (neat, $\mathrm{cm}^{-1}$ ): $v_{\mathrm{SCN}}=2157, v_{\mathrm{C}=\mathrm{O}}=1736$. MS (ES, pos. mode) $m / z$ (\%): $243\left(\mathrm{M}+\mathrm{H}^{+}, 100\right)$. Anal. Calcd for $\mathrm{C}_{11} \mathrm{H}_{18} \mathrm{~N}_{2} \mathrm{O}_{2} \mathrm{~S}(\%)$ : C, 54.52; $\mathrm{H}$, 7.49; N, 11.56. Found (\%): C, 54.27; H, 7.64; N, 11.64.

Ethyl 1-tert-pentyl-3-thiocyanoazetidine-3-carboxylate (22b)

Yellow oil, yield 83\%, $\mathrm{R}_{f}=0.21$ (petroleum ether/Et ${ }_{2} \mathrm{O}$ 7/3). ${ }^{1} \mathrm{H} \mathrm{NMR}(300 \mathrm{MHz}$, $\left.\mathrm{CDCl}_{3}\right): \delta 0.85\left(3 \mathrm{H}, \mathrm{t}, J=7.4 \mathrm{~Hz}, \mathrm{CCH}_{2} \mathrm{CH}_{3}\right), 0.90\left(6 \mathrm{H}, \mathrm{s}, \mathrm{C}\left(\mathrm{CH}_{3}\right)_{2}\right), 1.25(2 \mathrm{H}, \mathrm{q}$, $\left.J=7.7 \mathrm{~Hz}, \mathrm{CCH}_{2} \mathrm{CH}_{3}\right), 1.35\left(3 \mathrm{H}, \mathrm{t}, J=7.2 \mathrm{~Hz}, \mathrm{OCH}_{2} \mathrm{CH}_{3}\right), 3.43(2 \mathrm{H}, \mathrm{d}, J=8.8$ $\mathrm{Hz}, \mathrm{CH}(\mathrm{H}) \mathrm{NCH}(\mathrm{H})), 3.82(2 \mathrm{H}, \mathrm{d}, J=9.4 \mathrm{~Hz}, \mathrm{CH}(H) \mathrm{NCH}(H)), 4.31(2 \mathrm{H}, \mathrm{q}, J=$ $\left.7.2 \mathrm{~Hz}, \mathrm{OCH}_{2} \mathrm{CH}_{3}\right) .{ }^{13} \mathrm{C} \mathrm{NMR}\left(75 \mathrm{MHz}, \mathrm{CDCl}_{3}\right): \delta 8.5,14.1,20.2,31.4,47.9$, 54.8, 55.6, 63.0, 110.0, 169.3. IR (neat, $\left.\mathrm{cm}^{-1}\right): v_{\mathrm{SCN}}=2157, v_{\mathrm{C}=\mathrm{O}}=1736$. MS $(\mathrm{ES}$, pos. mode) $m / z(\%): 257\left(\mathrm{M}+\mathrm{H}^{+}, 100\right)$. Anal. Calcd for $\mathrm{C}_{12} \mathrm{H}_{20} \mathrm{~N}_{2} \mathrm{O}_{2} \mathrm{~S}(\%): \mathrm{C}$, 56.22; H, 7.86; N, 10.93. Found (\%): C, 56.09; H, 8.01; N, 10.64.

Synthesis of alkyl 3-azidoazetidine-3-carboxylates 23a-c

As a representative example, the synthesis of ethyl 1-tert-pentyl-3-azidoazetidine3-carboxylate (23b) is described here. A solution of ethyl 1-tert-pentyl-3bromoazetidine-3-carboxylate $(\mathbf{1 5 b})(111 \mathrm{mg}, 0.4 \mathrm{mmol})$ and sodium azide (52 $\mathrm{mg}, 0.8 \mathrm{mmol})$ in DMSO $(2 \mathrm{~mL})$ was heated at $60{ }^{\circ} \mathrm{C}$ for $3 \mathrm{~h}$. After cooling, the reaction mixture was poured into water $(15 \mathrm{~mL})$ and extracted with $\mathrm{Et}_{2} \mathrm{O}(3 \times 15$ $\mathrm{mL})$. The combined organic extracts were washed with brine $(2 \times 15 \mathrm{~mL})$ and water $(10 \mathrm{~mL})$. Drying $\left(\mathrm{MgSO}_{4}\right)$, filtration, evaporation of the solvent under reduced pressure and purification by preparative TLC on silica gel (petroleum ether/ $\mathrm{Et}_{2} \mathrm{O} 8 / 2$ ) afforded 3-azidoazetidine $23 \mathbf{b}$ in $98 \%$ yield. 
Yellow oil, yield 86\%, $\mathrm{R}_{f}=0.18$ (petroleum ether/Et $\mathrm{E}_{2} \mathrm{O}$ 7/3). ${ }^{1} \mathrm{H}$ NMR (300 MHz, $\left.\mathrm{CDCl}_{3}\right): \delta 0.99\left(9 \mathrm{H}, \mathrm{s}, \mathrm{C}\left(\mathrm{CH}_{3}\right)_{3}\right), 1.34\left(3 \mathrm{H}, \mathrm{t}, J=7.2 \mathrm{~Hz}, \mathrm{CH}_{2} \mathrm{CH}_{3}\right), 3.32(2 \mathrm{H}, \mathrm{d}, J$ $=7.4 \mathrm{~Hz}, \mathrm{CH}(\mathrm{H}) \mathrm{NCH}(\mathrm{H})), 3.70(2 \mathrm{H}, \mathrm{d}, J=7.4 \mathrm{~Hz}, \mathrm{CH}(H) \mathrm{NCH}(H)), 4.29(2 \mathrm{H}, \mathrm{q}$, $\left.J=7.2 \mathrm{~Hz}, \mathrm{OCH}_{2} \mathrm{CH}_{3}\right) .{ }^{13} \mathrm{C} \mathrm{NMR}\left(75 \mathrm{MHz}, \mathrm{CDCl}_{3}\right): \delta$ 14.2, 24.0, 52.2, 54.4, 59.3, 62.4, 170.0. IR (neat, $\left.\mathrm{cm}^{-1}\right): v_{\mathrm{N} 3}=2109, v_{\mathrm{C}=\mathrm{O}}=1737$. MS (ES, pos. mode): $m / z(\%): 227\left(\mathrm{M}+\mathrm{H}^{+}, 100\right)$. Anal. Calcd for $\mathrm{C}_{10} \mathrm{H}_{18} \mathrm{~N}_{4} \mathrm{O}_{2}(\%)$ : C, 53.08; $\mathrm{H}, 8.02$; N, 24.76. Found (\%): C, 52.74; H, 8.31; N, 24.90.

Ethyl 1-tert-pentyl-3-azidoazetidine-3-carboxylate (23b)

Yellow oil, yield 98\%, $\mathrm{R}_{f}=0.24$ (petroleum ether/Et ${ }_{2} \mathrm{O}$ 8/2). ${ }^{1} \mathrm{H}$ NMR (300 MHz, $\left.\mathrm{CDCl}_{3}\right): \delta 0.85\left(3 \mathrm{H}, \mathrm{t}, J=7.4 \mathrm{~Hz}, \mathrm{CCH}_{2} \mathrm{CH}_{3}\right), 0.91\left(6 \mathrm{H}, \mathrm{s}, \mathrm{C}\left(\mathrm{CH}_{3}\right)_{2}\right), 1.27(2 \mathrm{H}, \mathrm{q}$, $\left.J=7.4 \mathrm{~Hz}, \mathrm{CCH}_{2} \mathrm{CH}_{3}\right), 1.34\left(3 \mathrm{H}, \mathrm{t}, J=7.2 \mathrm{~Hz}, \mathrm{OCH}_{2} \mathrm{CH}_{3}\right), 3.30(2 \mathrm{H}, \mathrm{dd}, J=7.2$ $\mathrm{Hz}, 1.4 \mathrm{~Hz}, \mathrm{CH}(\mathrm{H}) \mathrm{NCH}(\mathrm{H})), 3.70(2 \mathrm{H}, \mathrm{dd}, J=7.3 \mathrm{~Hz}, 1.4 \mathrm{~Hz}, \mathrm{CH}(H) \mathrm{NCH}(H))$, $4.28\left(2 \mathrm{H}, \mathrm{q}, J=7.2 \mathrm{~Hz}, \mathrm{OCH}_{2} \mathrm{CH}_{3}\right) .{ }^{13} \mathrm{C} \mathrm{NMR}\left(75 \mathrm{MHz}, \mathrm{CDCl}_{3}\right): \delta 8.6,14.2$, 20.0, 31.5, 54.3, 54.6, 59.7, 62.3, 170.0. IR (neat, $\left.\mathrm{cm}^{-1}\right): v_{\mathrm{N} 3}=2108, v_{\mathrm{C}=\mathrm{O}}=1738$. MS (ES, pos. mode): $m / z(\%): 241\left(\mathrm{M}+\mathrm{H}^{+}, 100\right)$. Anal. Calcd for $\mathrm{C}_{11} \mathrm{H}_{20} \mathrm{~N}_{4} \mathrm{O}_{2}$ (\%): C, 54.98; H, 8.39; N, 23.32. Found (\%): C, 54.59; H, 8.53; N, 23.64.

Methyl 1-tert-butyl-3-azidoazetidine-3-carboxylate (23c)

Yellow oil, yield 79\%, $\mathrm{R}_{f}=0.22$ (petroleum ether/EtOAc 7/3). ${ }^{1} \mathrm{H}$ NMR (300 $\left.\mathrm{MHz}, \mathrm{CDCl}_{3}\right): \delta 0.99\left(9 \mathrm{H}, \mathrm{s}, \mathrm{C}\left(\mathrm{CH}_{3}\right)_{3}\right), 3.33(2 \mathrm{H}, \mathrm{d}, J=7.4 \mathrm{~Hz}, \mathrm{CH}(\mathrm{H}) \mathrm{NCH}(\mathrm{H}))$, $3.70(2 \mathrm{H}, \mathrm{d}, J=7.4 \mathrm{~Hz}, \mathrm{CH}(H) \mathrm{NCH}(H)), 3.84\left(3 \mathrm{H}, \mathrm{s}, \mathrm{OCH}_{3}\right) .{ }^{13} \mathrm{C} \mathrm{NMR}(75 \mathrm{MHz}$, $\left.\mathrm{CDCl}_{3}\right): \delta 24.0,52.2,53.2,54.5,59.4,170.5$. IR (neat, $\left.\mathrm{cm}^{-1}\right): v_{\mathrm{N} 3}=2109, v_{\mathrm{C}=\mathrm{O}}=$ 1740. MS (ES, pos. mode): $m / z(\%): 213\left(\mathrm{M}+\mathrm{H}^{+}, 100\right)$. Anal. Calcd for $\mathrm{C}_{9} \mathrm{H}_{16} \mathrm{~N}_{4} \mathrm{O}_{2}$ (\%): C, 50.93; H, 7.60; N, 26.40. Found (\%): C, 50.79; H, 7.69; N, 26.21 .

Synthesis of alkyl 3-phenoxyazetidine-3-carboxylates 20a-b 
As a representative example, the synthesis of ethyl 1-tert-pentyl-3phenoxyazetidine-3-carboxylate (20b) is described here. Ethyl 1-tert-pentyl-3bromoazetidine-3-carboxylate $(\mathbf{1 5 b})(83 \mathrm{mg}, 0.3 \mathrm{mmol})$ was added to a mixture of phenol (70 mg, $0.75 \mathrm{mmol}$ ) and $\mathrm{K}_{2} \mathrm{CO}_{3}(206 \mathrm{mg}, 1.5 \mathrm{mmol})$ in DMSO (2 mL) and the reaction mixture was heated at $60{ }^{\circ} \mathrm{C}$ for $8 \mathrm{~h}$. After cooling, the reaction mixture was poured into water $(15 \mathrm{~mL})$ and extracted with $\mathrm{Et}_{2} \mathrm{O}(3 \times 15 \mathrm{~mL})$. The combined organic extracts were washed with brine $(2 \times 15 \mathrm{~mL})$, saturated $\mathrm{NaOH}$ (aq.) solution $(2 \times 10 \mathrm{~mL})$ and water $(15 \mathrm{~mL})$. Drying $\left(\mathrm{MgSO}_{4}\right)$, filtration, evaporation of the solvent under reduced pressure and purification by preparative TLC on silica gel (petroleum ether/ $\mathrm{Et}_{2} \mathrm{O} 7 / 3$ ) afforded 3-phenoxyazetidine $20 \mathrm{~b}$ in $81 \%$ yield.

Ethyl 1-tert-butyl-3-phenoxyazetidine-3-carboxylate (20a)

Yellow oil, yield 36\%, $\mathrm{R}_{f}=0.25$ (petroleum ether/Et ${ }_{2} \mathrm{O}$ 1/1). ${ }^{1} \mathrm{H}$ NMR (300 MHz, $\left.\mathrm{CDCl}_{3}\right): \delta 1.00\left(9 \mathrm{H}, \mathrm{s}, \mathrm{C}\left(\mathrm{CH}_{3}\right)_{3}\right), 1.15\left(3 \mathrm{H}, \mathrm{t}, J=7.2 \mathrm{~Hz}, \mathrm{CH}_{2} \mathrm{CH}_{3}\right), 3.51(2 \mathrm{H}, \mathrm{d}, J$ $=9.1 \mathrm{~Hz}, \mathrm{CH}(\mathrm{H}) \mathrm{NCH}(\mathrm{H})), 3.88(2 \mathrm{H}, \mathrm{d}, J=9.1 \mathrm{~Hz}, \mathrm{CH}(H) \mathrm{NCH}(H)), 4.21(2 \mathrm{H}, \mathrm{q}$, $\left.J=7.2 \mathrm{~Hz}, \mathrm{OCH}_{2} \mathrm{CH}_{3}\right), 6.65-6.69\left(2 \mathrm{H}, \mathrm{m}, 2 \times \mathrm{CH}_{\text {arom }}\right), 6.93-6.98\left(1 \mathrm{H}, \mathrm{m}, \mathrm{CH}_{\text {arom }}\right)$, 7.20-7.27 (2H, m, $\left.2 \times \mathrm{CH}_{\text {arom }}\right) .{ }^{13} \mathrm{C} \mathrm{NMR}\left(75 \mathrm{MHz}, \mathrm{CDCl}_{3}\right): \delta 14.1,24.1,52.2$, 55.0, 61.9, 73.8, 115.2, 121.5, 129.6, 155.4, 171.4. IR (neat, $\mathrm{cm}^{-1}$ ): $v_{\mathrm{C}=\mathrm{O}}=1736$. MS (ES, pos. mode): $m / z$ (\%): $278\left(\mathrm{M}+\mathrm{H}^{+}, 100\right)$. Anal. Calcd for $\mathrm{C}_{16} \mathrm{H}_{23} \mathrm{NO}_{3}(\%)$ : C, 69.29; H, 8.36; N, 5.05. Found (\%): C, 69.02; H, 8.59; N, 4.83.

Ethyl 1-tert-pentyl-3-phenoxyazetidine-3-carboxylate (20b)

Yellow oil, yield 81\%, $\mathrm{R}_{f}=0.27$ (petroleum ether/Et ${ }_{2} \mathrm{O} 7 / 3$ ). ${ }^{1} \mathrm{H}$ NMR (300 MHz, $\left.\mathrm{CDCl}_{3}\right): \delta 0.85\left(3 \mathrm{H}, \mathrm{t}, J=7.4 \mathrm{~Hz}, \mathrm{CCH}_{2} \mathrm{CH}_{3}\right), 0.92\left(6 \mathrm{H}, \mathrm{s}, \mathrm{C}\left(\mathrm{CH}_{3}\right)_{2}\right), 1.15(3 \mathrm{H}, \mathrm{t}$, $\left.J=7.2 \mathrm{~Hz}, \mathrm{OCH}_{2} \mathrm{CH}_{3}\right), 1.30\left(2 \mathrm{H}, \mathrm{q}, J=7.4 \mathrm{~Hz}, \mathrm{CCH}_{2} \mathrm{CH}_{3}\right), 3.51(2 \mathrm{H}, \mathrm{d}, J=8.0$ $\mathrm{Hz}, \mathrm{CH}(\mathrm{H}) \mathrm{NCH}(\mathrm{H})), 3.86(2 \mathrm{H}, \mathrm{d}, J=8.0 \mathrm{~Hz}, \mathrm{CH}(H) \mathrm{NCH}(H)), 4.21(2 \mathrm{H}, \mathrm{q}, J=$ $\left.7.2 \mathrm{~Hz}, \mathrm{OCH}_{2} \mathrm{CH}_{3}\right), 6.66-6.69\left(2 \mathrm{H}, \mathrm{m}, 2 \times \mathrm{CH}_{\text {arom }}\right), 6.93-6.98\left(1 \mathrm{H}, \mathrm{m}, \mathrm{CH}_{\text {arom }}\right)$, 7.21-7.26 (2H, m, $\left.2 \times \mathrm{CH}_{\text {arom }}\right) .{ }^{13} \mathrm{C} \mathrm{NMR}\left(75 \mathrm{MHz}, \mathrm{CDCl}_{3}\right): \delta 8.7,14.1,20.0,31.5$, $54.7,54.8,61.8,74.1,115.2,121.5,129.6,155.5,171.4$. IR $\left(\right.$ neat, $\left.\mathrm{cm}^{-1}\right): v_{\mathrm{C}=\mathrm{O}}=$ 1736. MS (ES, pos. mode): $m / z(\%): 292\left(\mathrm{M}+\mathrm{H}^{+}, 100\right)$. Anal. Calcd for 
$\mathrm{C}_{17} \mathrm{H}_{25} \mathrm{NO}_{3}(\%): \mathrm{C}, 70.07 ; \mathrm{H}, 8.65 ; \mathrm{N}, 4.81$. Found (\%): C, 70.34; H, 8.40; N, 4.60 .

Synthesis of ethyl 1-tert-pentyl-2-(aminomethyl)aziridine-2-carboxylate (24b)

To a solution of ethyl 1-tert-pentyl-2-(azidomethyl)aziridine-2-carboxylate (18b) (70 mg, $0.29 \mathrm{mmol})$ in EtOH $(7 \mathrm{~mL}), \mathrm{Pd}(7 \mathrm{mg}, 10 \mathrm{wt} \%$ on carbon) was added and the reaction mixture was stirred under $\mathrm{H}_{2}$ atmosphere (4 bar) at room temperature for $3 \mathrm{~h}$. Subsequently, the reaction mixture was filtered over Celite ${ }^{\circledR}$ and the filter cake was washed with small portions of EtOH. Evaporation of the solvent under reduced pressure and purification by preparative TLC on silica gel $\left(\mathrm{CH}_{2} \mathrm{Cl}_{2} / \mathrm{MeOH} 100 / 5\right)$ afforded 2-(aminomethyl)aziridine 24b. Yellow oil, yield $68 \%, \mathrm{R}_{f}=0.27\left(\mathrm{CH}_{2} \mathrm{Cl}_{2} / \mathrm{MeOH} 9 / 1\right) .{ }^{1} \mathrm{H} \mathrm{NMR}\left(300 \mathrm{MHz}, \mathrm{CDCl}_{3}\right): \delta 0.92(3 \mathrm{H}, \mathrm{t}, J$ $\left.=7.6 \mathrm{~Hz}, \mathrm{CCH}_{2} \mathrm{CH}_{3}\right), 0.94\left(3 \mathrm{H}, \mathrm{s}, \mathrm{CCH}_{3}\right), 0.98\left(3 \mathrm{H}, \mathrm{s}, \mathrm{CCH}_{3}\right), 1.30(3 \mathrm{H}, \mathrm{t}, J=7.2$ $\left.\mathrm{Hz}, \mathrm{OCH}_{2} \mathrm{CH}_{3}\right), 1.48\left(1 \mathrm{H}, \mathrm{dq}, J=13.6 \mathrm{~Hz}, 7.4 \mathrm{~Hz}, \mathrm{CCH}(\mathrm{H}) \mathrm{CH}_{3}\right), 1.51(1 \mathrm{H}, \mathrm{dq}, J$ $\left.=13.4 \mathrm{~Hz}, 7.4 \mathrm{~Hz}, \mathrm{CCH}(H) \mathrm{CH}_{3}\right), 1.78(1 \mathrm{H}, \mathrm{d}, J=1.1 \mathrm{~Hz}, \mathrm{CH}(\mathrm{H}) \mathrm{N}), 1.86(2 \mathrm{H}, \mathrm{br}$ s, $\left.\mathrm{NH}_{2}\right), 2.41(1 \mathrm{H}, \mathrm{d}, J=1.4 \mathrm{~Hz}, \mathrm{CH}(H) \mathrm{N}), 2.87\left(1 \mathrm{H}, \mathrm{d}, J=13.8 \mathrm{~Hz}, \mathrm{CH}(\mathrm{H}) \mathrm{NH}_{2}\right)$, $2.89\left(1 \mathrm{H}, \mathrm{d}, J=13.8 \mathrm{~Hz}, \mathrm{CH}(H) \mathrm{NH}_{2}\right), 4.16(1 \mathrm{H}, \mathrm{dq}, J=10.7 \mathrm{~Hz}, 7.2 \mathrm{~Hz}$, $\left.\mathrm{OCH}(\mathrm{H}) \mathrm{CH}_{3}\right), 4.22\left(1 \mathrm{H}, \mathrm{dq}, J=10.7 \mathrm{~Hz}, 7.2 \mathrm{~Hz}, \mathrm{OCH}(H) \mathrm{CH}_{3}\right) .{ }^{13} \mathrm{C} \mathrm{NMR}(75$ $\mathrm{MHz}, \mathrm{CDCl}_{3}$ ): $\delta 8.9,14.1,23.8,24.5,31.4,36.7,46.9,56.5,61.3,172.1$. IR (neat, $\left.\mathrm{cm}^{-1}\right): v_{\mathrm{NH} 2}=3381($ weak $), v_{\mathrm{C}=\mathrm{O}}=1724$. MS $(\mathrm{ES}$, pos. mode $): \mathrm{m} / z(\%): 215$ $\left(\mathrm{M}+\mathrm{H}^{+}, 100\right)$. Anal. Calcd for $\mathrm{C}_{11} \mathrm{H}_{22} \mathrm{~N}_{2} \mathrm{O}_{2}(\%)$ : C, 61.65; H, 10.35; N, 13.07. Found (\%): C, 61.27; H, 10.62; N, 12.91.

Synthesis of alkyl 2-(tert-butoxycarbonylaminomethyl)aziridine-2-carboxylates 25b-c

As a representative example, the synthesis of methyl 1-tert-butyl-2-(tertbutoxycarbonylaminomethyl)aziridine-2-carboxylate (25c) is described here. To a solution of methyl 1-tert-butyl-2-(azidomethyl)aziridine-2-carboxylate (18c) (65 $\mathrm{mg}, 0.31 \mathrm{mmol})$ in degassed $\mathrm{CH}_{3} \mathrm{CN}(8 \mathrm{~mL}), \mathrm{Pd}(6.5 \mathrm{mg}, 10 \mathrm{wt} \%$ on carbon) and di-tert-butyl dicarbonate $(334 \mathrm{mg}, 1.53 \mathrm{mmol})$ were added and the reaction mixture was stirred under $\mathrm{H}_{2}$ atmosphere (10 bar) at room temperature for $3 \mathrm{~h}$. Subsequently, the reaction mixture was filtered over Celite ${ }^{\circledR}$ and the filter cake 
was washed with small portions of $\mathrm{CH}_{3} \mathrm{CN}$. Evaporation of the solvent under reduced pressure and purification by flash chromatography on silica gel (hexane/EtOAc 9/1) afforded aziridine $\mathbf{2 5 c}$ in $76 \%$ yield.

Ethyl 1-tert-pentyl-2-(tert-butoxycarbonylaminomethyl)aziridine-2-carboxylate $(25 b)$

Light yellow oil, yield 49\%, $\mathrm{R}_{f}=0.13$ (hexane/EtOAc 9/1). ${ }^{1} \mathrm{H}$ NMR (300 MHz, $\left.\mathrm{CDCl}_{3}\right): \delta 0.91\left(3 \mathrm{H}, \mathrm{t}, J=7.7 \mathrm{~Hz}, \mathrm{CCH}_{2} \mathrm{CH}_{3}\right), 0.96\left(3 \mathrm{H}, \mathrm{s}, \mathrm{NCCH}_{3}\right), 0.97(3 \mathrm{H}, \mathrm{s}$, $\left.\mathrm{CCH}_{3}\right), 1.29\left(3 \mathrm{H}, \mathrm{t}, J=7.2 \mathrm{~Hz}, \mathrm{OCH}_{2} \mathrm{CH}_{3}\right), 1.40-1.50\left(2 \mathrm{H}, \mathrm{m}, \mathrm{CCH}_{2} \mathrm{CH}_{3}\right), 1.43$ $\left(9 \mathrm{H}, \mathrm{s}, \mathrm{NCCH}_{3}\right), 1.86(1 \mathrm{H}, \mathrm{d}, J=1.1 \mathrm{~Hz}, \mathrm{NCH}(\mathrm{H})), 2.39(1 \mathrm{H}, \mathrm{s}, \mathrm{NCH}(H)), 3.30$ $(1 \mathrm{H}, \mathrm{dd}, J=13.8 \mathrm{~Hz}, 4.4 \mathrm{~Hz}, \mathrm{CH}(\mathrm{H}) \mathrm{NH}), 3.60(1 \mathrm{H}, \mathrm{d}, J=13.8 \mathrm{~Hz}, 7.7 \mathrm{~Hz}$, $\mathrm{CH}(H) \mathrm{NH}), 4.18\left(2 \mathrm{H}, \mathrm{m}, \mathrm{OCH}_{2} \mathrm{CH}_{3}\right), 4.90(1 \mathrm{H}$, br s, $\mathrm{NH}) .{ }^{13} \mathrm{C} \mathrm{NMR}(75 \mathrm{MHz}$, $\left.\mathrm{CDCl}_{3}\right): \delta 8.8,14.0,24.2,24.6,28.5,31.0,36.7,42.3,43.5,56.6,61,4,79.3$, 155.9, 171.6. IR (neat, $\left.\mathrm{cm}^{-1}\right): v_{\mathrm{NH}}=3382, v_{\mathrm{C}=\mathrm{O}}=1717$. MS (ES, pos. mode) $\mathrm{m} / \mathrm{z}$ (\%): $315\left(\mathrm{M}+\mathrm{H}^{+}, 100\right)$. Anal. Calcd for $\mathrm{C}_{16} \mathrm{H}_{30} \mathrm{~N}_{2} \mathrm{O}_{4}(\%)$ : C, 61.12; H, 9.62; N, 8.91. Found (\%): C, 61.39; H, 9.51; N, 8.86.

Methyl 1-tert-butyl-2-(tert-butoxycarbonylaminomethyl)aziridine-2-carboxylate $(25 c)$

Light yellow oil, yield 76\%, $\mathrm{R}_{f}=0.07$ (hexane/EtOAc 9/1). ${ }^{1} \mathrm{H}$ NMR (300 MHz, $\left.\mathrm{CDCl}_{3}\right): \delta 1.07\left(9 \mathrm{H}, \mathrm{s}, \mathrm{NC}\left(\mathrm{CH}_{3}\right)_{3}\right), 1.44\left(9 \mathrm{H}, \mathrm{s}, \mathrm{OC}\left(\mathrm{CH}_{3}\right)_{3}\right), 1.87(1 \mathrm{H}, \mathrm{d}, J=1.1$ $\mathrm{Hz}, \mathrm{NCH}(\mathrm{H})), 2.34(1 \mathrm{H}, \mathrm{s}, \mathrm{NCH}(H)), 3.33(1 \mathrm{H}, \mathrm{dd}, J=13.8 \mathrm{~Hz}, 4.4 \mathrm{~Hz}$, $\mathrm{CH}(\mathrm{H}) \mathrm{NH}), 3.57(1 \mathrm{H}, \mathrm{dd}, J=13.8 \mathrm{~Hz}, 7.2 \mathrm{~Hz}, \mathrm{CH}(\mathrm{H}) \mathrm{NH}), 3.72\left(3 \mathrm{H}, \mathrm{s}, \mathrm{OCH}_{3}\right)$, $4.93(1 \mathrm{H}$, br s, $\mathrm{NH}) .{ }^{13} \mathrm{C} \mathrm{NMR}\left(75 \mathrm{MHz}, \mathrm{CDCl}_{3}\right): \delta$ 28.4, 28.5, 31.0, 42.9, 43.6, 52.4, 54.3, 79.3, 155.9, 172.0. IR (neat, $\left.\mathrm{cm}^{-1}\right): v_{\mathrm{NH}}=3382, v_{\mathrm{C}=\mathrm{O}}=1718$. MS $(\mathrm{ES}$, pos. mode): $m / z(\%): 287\left(\mathrm{M}+\mathrm{H}^{+}, 100\right)$. Anal. Calcd for $\mathrm{C}_{14} \mathrm{H}_{26} \mathrm{~N}_{2} \mathrm{O}_{4}(\%)$ : C, $58.72 ; \mathrm{H}, 9.15 ; \mathrm{N}, 9.78$. Found (\%): C, 58.59; H, 9.38; N, 9.59.

Synthesis of ethyl 1-tert-butyl-3-aminoazetidine-3-carboxylate (26a)

To a solution of ethyl 1-tert-butyl-3-azidoazetidine-3-carboxylate (23a) (52 mg, $0.23 \mathrm{mmol})$ in EtOH $(5 \mathrm{~mL}), \mathrm{Pd}(5.2 \mathrm{mg}, 10 \mathrm{wt} \%$ on carbon) was added and the 
reaction mixture was stirred under $\mathrm{H}_{2}$ atmosphere ( 4 bar) at room temperature for $3 \mathrm{~h}$. Subsequently, the reaction mixture was filtered over Celite ${ }^{\circledR}$ and the filter cake was washed with small portions of EtOH. Evaporation of the solvent under reduced pressure and purification by preparative TLC on silica gel (petroleum ether/EtOAc 1/1) afforded 3-aminoazetidine 26a in 78\% yield. Yellow oil, $\mathrm{R}_{f}=$ 0.10 (petroleum ether/EtOAc 1/1). ${ }^{1} \mathrm{H}$ NMR $\left(300 \mathrm{MHz}, \mathrm{CDCl}_{3}\right): \delta 0.99(9 \mathrm{H}, \mathrm{s}$, $\left.\mathrm{C}\left(\mathrm{CH}_{3}\right)_{3}\right), 1.31\left(3 \mathrm{H}, \mathrm{t}, J=7.2 \mathrm{~Hz}, \mathrm{CH}_{2} \mathrm{CH}_{3}\right), 2.37\left(2 \mathrm{H}\right.$, br s, $\left.\mathrm{NH}_{2}\right), 3.10(2 \mathrm{H}, \mathrm{d}, J=$ $8.3 \mathrm{~Hz}, \mathrm{NCH}(\mathrm{H})), 3.72(2 \mathrm{H}, \mathrm{d}, J=8.3 \mathrm{~Hz}, \mathrm{NCH}(H)), 4.22(2 \mathrm{H}, \mathrm{q}, J=7.2 \mathrm{~Hz}$, $\left.\mathrm{OCH}_{2} \mathrm{CH}_{3}\right) .{ }^{13} \mathrm{C}$ NMR $\left(75 \mathrm{MHz}, \mathrm{CDCl}_{3}\right): \delta 14.3,24.0,52.2,53.1,57.7,61.5$, 173.9. IR (neat, $\left.\mathrm{cm}^{-1}\right): v_{\mathrm{NH} 2}=3376$ (weak), $v_{\mathrm{C}=\mathrm{O}}=1730$. MS (ES, pos. mode) $: \mathrm{m} / \mathrm{z}$ (\%): $201\left(\mathrm{M}+\mathrm{H}^{+}\right.$, 100). Anal. Calcd for $\mathrm{C}_{10} \mathrm{H}_{20} \mathrm{~N}_{2} \mathrm{O}_{2}(\%)$ : C, 59.97; H, 10.07; N, 13.99. Found (\%): C, 59.61; H, 10.17; N, 13.64.

Synthesis of alkyl 3-azidoazetidine-3-carboxylic acids $\mathbf{2 7 a - b}$

As a representative example, the synthesis of 1-tert-butyl-3-azidoazetidine-3carboxylic acid (27a) is described here. To a solution of ethyl 1-tert-butyl-3azidoazetidine-3-carboxylate (23a) $(96 \mathrm{mg}, 0.42 \mathrm{mmol})$ in $\mathrm{MeOH}(3 \mathrm{~mL}), 2 \mathrm{~N}$ $\mathrm{NaOH}$ (aq.) solution ( $3 \mathrm{~mL}$ ) was added dropwise at $0{ }^{\circ} \mathrm{C}$ and the reaction mixture was stirred at room temperature for $3 \mathrm{~h}$. The reaction mixture was acidified with $1 \mathrm{~N} \mathrm{HCl}$ aq. solution till $\mathrm{pH}=7$ and the solvents were evaporated under reduced pressure. The residue was dissolved in $\mathrm{H}_{2} \mathrm{O}$ and purified by means of ionexchange chromatography on Dowex $\mathrm{H}^{+}(50 \times 8-100)$ by sequential elution with $\mathrm{H}_{2} \mathrm{O}$ and $1 \mathrm{~N} \mathrm{NH}_{4} \mathrm{OH}$ aq. solution. The eluted aqueous solution of the ammonium salt was neutralized with $2 \mathrm{~N} \mathrm{HCl}$ aq. solution and the solvent was evaporated under reduced pressure. The residue was taken up in $\mathrm{CH}_{2} \mathrm{Cl}_{2}$, filtered and evaporated under reduced pressure to afford carboxylic acid $\mathbf{2 7}$ a in $90 \%$ yield.

1-tert-Butyl-3-azidoazetidine-3-carboxylic acid (27a)

Yellow viscous oil, yield 90\%, $\mathrm{R}_{f}=0.15\left(\mathrm{CH}_{2} \mathrm{Cl}_{2} / \mathrm{MeOH} 9 / 1\right) .{ }^{1} \mathrm{H}$ NMR (300 $\left.\mathrm{MHz}, \quad \mathrm{CD}_{3} \mathrm{OD}\right): \quad \delta 1.34\left(9 \mathrm{H}, \quad \mathrm{s}, \mathrm{C}\left(\mathrm{CH}_{3}\right)_{3}\right), 4.05(2 \mathrm{H}, \mathrm{d}, J=11.0 \mathrm{~Hz}$, $\mathrm{CH}(\mathrm{H}) \mathrm{NCH}(\mathrm{H})), 4.51(2 \mathrm{H}, \mathrm{d}, J=11.6 \mathrm{~Hz}, \mathrm{CH}(H) \mathrm{NCH}(H)) .{ }^{13} \mathrm{C} \mathrm{NMR}(75 \mathrm{MHz}$, $\left.\mathrm{CD}_{3} \mathrm{OD}\right): \delta 24.2,53.5,55.6,62.2,176.5$. IR $\left(\right.$ neat, $\left.\mathrm{cm}^{-1}\right): v_{\mathrm{OH}}=3425, v_{\mathrm{N} 3}=2121$, 
$v_{\mathrm{C}=\mathrm{O}}=1623 . \mathrm{MS}(\mathrm{ES}$, pos. mode $): \mathrm{m} / \mathrm{z}(\%): 199\left(\mathrm{M}+\mathrm{H}^{+}, 100\right)$. Anal. Calcd for $\mathrm{C}_{8} \mathrm{H}_{14} \mathrm{~N}_{4} \mathrm{O}_{2}$ (\%): C, 48.47; H, 7.12; N, 28.26. Found (\%): C, 48.09; H, 6.98; N, 28.07.

1-tert-Pentyl-3-azidoazetidine-3-carboxylic acid (27b)

Yellow viscous oil, yield 76\%, $\mathrm{R}_{f}=0.21\left(\mathrm{CH}_{2} \mathrm{Cl}_{2} / \mathrm{MeOH} 9 / 1\right) .{ }^{1} \mathrm{H}$ NMR (300 $\left.\mathrm{MHz}, \mathrm{CD}_{3} \mathrm{OD}\right): \delta 0.98\left(3 \mathrm{H}, \mathrm{t}, J=7.7 \mathrm{~Hz}, \mathrm{CCH}_{2} \mathrm{CH}_{3}\right), 1.30\left(6 \mathrm{H}, \mathrm{s}, \mathrm{C}\left(\mathrm{CH}_{3}\right)_{2}\right), 1.27$ $\left(2 \mathrm{H}, \mathrm{q}, J=7.7 \mathrm{~Hz}, \mathrm{CCH}_{2} \mathrm{CH}_{3}\right), 4.07(2 \mathrm{H}, \mathrm{d}, J=11.0 \mathrm{~Hz}, \mathrm{CH}(\mathrm{H}) \mathrm{NCH}(\mathrm{H})), 4.54$ $(2 \mathrm{H}, \mathrm{d}, J=11.6 \mathrm{~Hz}, \mathrm{CH}(H) \mathrm{NCH}(H)) .{ }^{13} \mathrm{C} \mathrm{NMR}\left(75 \mathrm{MHz}, \mathrm{CD}_{3} \mathrm{OD}\right): \delta 8.6,20.0$, 31.5, 54.3, 54.6, 59.7, 170.0. IR (neat, $\mathrm{cm}^{-1}$ ): $v_{\mathrm{OH}}=3417, v_{\mathrm{N} 3}=2102, v_{\mathrm{C}=\mathrm{O}}=1604$. MS (ES, pos. mode): $m / z(\%): 213\left(\mathrm{M}+\mathrm{H}^{+}, 100\right)$. Anal. Calcd for $\mathrm{C}_{9} \mathrm{H}_{16} \mathrm{~N}_{4} \mathrm{O}_{2}(\%)$ : C, 50.93; H, 7.60; N, 26.40. Found (\%): C, 50.74; H, 7.78; N, 26.29.

Synthesis of alkyl 3-aminoazetidine-3-carboxylic acids 28a-b

As a representative example, the synthesis of 1-tert-pentyl-3-aminoazetidine-3carboxylic acid (28b) is described here. To a solution of 1-tert-pentyl-3azidoazetidine-3-carboxylic acid (27b) (60 mg, $0.3 \mathrm{mmol})$ in $\mathrm{MeOH}(12 \mathrm{~mL}), \mathrm{Pd}$ (6 $\mathrm{mg}, 10 \mathrm{wt} \%$ on carbon) was added and the reaction mixture was stirred under $\mathrm{H}_{2}$ atmosphere ( 3 bar) at room temperature for $3 \mathrm{~h}$. Subsequently, the reaction mixture was filtered over Celite ${ }^{\circledR}$ and the filter cake was washed with small portions of $\mathrm{MeOH}$. The solvent was evaporated under reduced pressure. The residue was dissolved in $\mathrm{H}_{2} \mathrm{O}$ and purified by means of ion-exchange chromatography on Dowex $\mathrm{H}^{+}(50 \times 8-100)$ by sequential elution with $\mathrm{H}_{2} \mathrm{O}$ and $1 \mathrm{~N} \mathrm{NH}_{4} \mathrm{OH}$ aq. solution. The eluted aqueous solution of the ammonium salt was neutralized with $2 \mathrm{~N} \mathrm{HCl}$ aq. solution and the solvent was evaporated under reduced pressure. The residue was taken up in $\mathrm{CH}_{2} \mathrm{Cl}_{2}$, filtered and evaporated under reduced pressure to afford amino acid $\mathbf{2 8 b}$ in $80 \%$ yield.

1-tert-Butyl-3-aminoazetidine-3-carboxylic acid (28a)

Brown viscous oil, yield $76 \%, \mathrm{R}_{f}=0.02\left(\mathrm{CH}_{2} \mathrm{Cl}_{2} / \mathrm{MeOH} 9 / 1\right) .{ }^{1} \mathrm{H}$ NMR (300 $\left.\mathrm{MHz}, \mathrm{CD}_{3} \mathrm{OD}\right): \delta 1.21\left(9 \mathrm{H}, \mathrm{s}, \mathrm{C}\left(\mathrm{CH}_{3}\right)_{3}\right), 3.75(2 \mathrm{H}, \mathrm{d}, J=10.5 \mathrm{~Hz}$, 
$\mathrm{CH}(\mathrm{H}) \mathrm{NCH}(\mathrm{H})), 4.25(2 \mathrm{H}, \mathrm{d}, J=10.5 \mathrm{~Hz}, \mathrm{CH}(H) \mathrm{NCH}(H)) .{ }^{13} \mathrm{C} \mathrm{NMR}(75 \mathrm{MHz}$, $\left.\mathrm{CD}_{3} \mathrm{OD}\right): \delta 23.5,54.2,59.1,59.3,176.4$. IR (neat, $\left.\mathrm{cm}^{-1}\right): v_{\mathrm{OH}, \mathrm{NH} 2}=3379, v_{\mathrm{C}=\mathrm{O}}=$ 1650. MS (ES, pos. mode): $m / z(\%): 173\left(\mathrm{M}+\mathrm{H}^{+}, 100\right)$. Anal. Calcd for $\mathrm{C}_{8} \mathrm{H}_{16} \mathrm{~N}_{2} \mathrm{O}_{2}$ (\%): C, 55.79; H, 9.36; N, 16.27. Found (\%): C, 55.51; H, 9.52; N, 16.39 .

1-tert-Pentyl-3-aminoazetidine-3-carboxylic acid (28b)

Brown viscous oil, yield 80\%, $\mathrm{R}_{f}=0.03\left(\mathrm{CH}_{2} \mathrm{Cl}_{2} / \mathrm{MeOH} 9 / 1\right) .{ }^{1} \mathrm{H}$ NMR (300 $\left.\mathrm{MHz}, \mathrm{CD}_{3} \mathrm{OD}\right): \delta 0.96\left(3 \mathrm{H}, \mathrm{t}, J=7.4 \mathrm{~Hz}, \mathrm{CCH}_{2} \mathrm{CH}_{3}\right), 1.21\left(6 \mathrm{H}, \mathrm{s}, \mathrm{C}\left(\mathrm{CH}_{3}\right)_{2}\right), 1.56$ $\left(2 \mathrm{H}, \mathrm{q}, J=7.4 \mathrm{~Hz}, \mathrm{CCH}_{2} \mathrm{CH}_{3}\right), 3.72(2 \mathrm{H}, \mathrm{d}, J=10.5 \mathrm{~Hz}, \mathrm{CH}(\mathrm{H}) \mathrm{NCH}(\mathrm{H})), 4.24$ $(2 \mathrm{H}, \mathrm{d}, J=9.9 \mathrm{~Hz}, \mathrm{CH}(H) \mathrm{NCH}(H)) .{ }^{13} \mathrm{C} \mathrm{NMR}\left(75 \mathrm{MHz}, \mathrm{CD}_{3} \mathrm{OD}\right): \delta 8.6,20.0$, $30.8,54.8,59.3,61.5,176.8$. IR (neat, $\mathrm{cm}^{-1}$ ): $v_{\mathrm{OH}, \mathrm{NH} 2}=3338, v_{\mathrm{C}=\mathrm{O}}=1625 . \mathrm{MS}$ (ES, pos. mode): $m / z(\%): 187\left(\mathrm{M}+\mathrm{H}^{+}, 100\right)$. Anal. Calcd for $\mathrm{C}_{9} \mathrm{H}_{18} \mathrm{~N}_{2} \mathrm{O}_{2}(\%): \mathrm{C}$, 58.04; H, 9.74; N, 15.04. Found (\%): C, 57.85; H, 9.87; N, 14.83.

\section{Results and discussion}

The synthesis of $N$-substituted alkyl 2-(aminomethyl)acrylates 12 via substitution reactions of alkyl 2-(bromomethyl)acrylates $\mathbf{1 1}$ with primary amines was performed under optimized conditions in analogy with literature procedures (Baraki et al. 1999; Habaue et al. 1997). Addition of alkyl 2(bromomethyl)acrylates $\mathbf{1 1}$ to a solution of primary amine and triethylamine in dichloromethane at $0 \quad{ }^{\circ} \mathrm{C}$ afforded ethyl and methyl 2[(alkylamino)methyl]acrylates 12 in excellent yields (Table 1). Subsequently, following a typical procedure for the bromination of functionalized allylamines (De Smaele et al. 2001), the amino group of alkyl 2[(alkylamino)methyl]acrylates $\mathbf{1 2}$ was protected by treatment with aqueous hydrobromic acid in dichloromethane to the corresponding hydrobromide salts. Subsequent bromination followed by neutralization of the reaction mixture with an aqueous $\mathrm{NaHCO}_{3}$ solution afforded the desired dibromopropanoates $\mathbf{1 3}$ in excellent yields (Table 1). 
Table 1 Synthesis and bromination of alkyl 2-(aminomethyl)acrylates 12

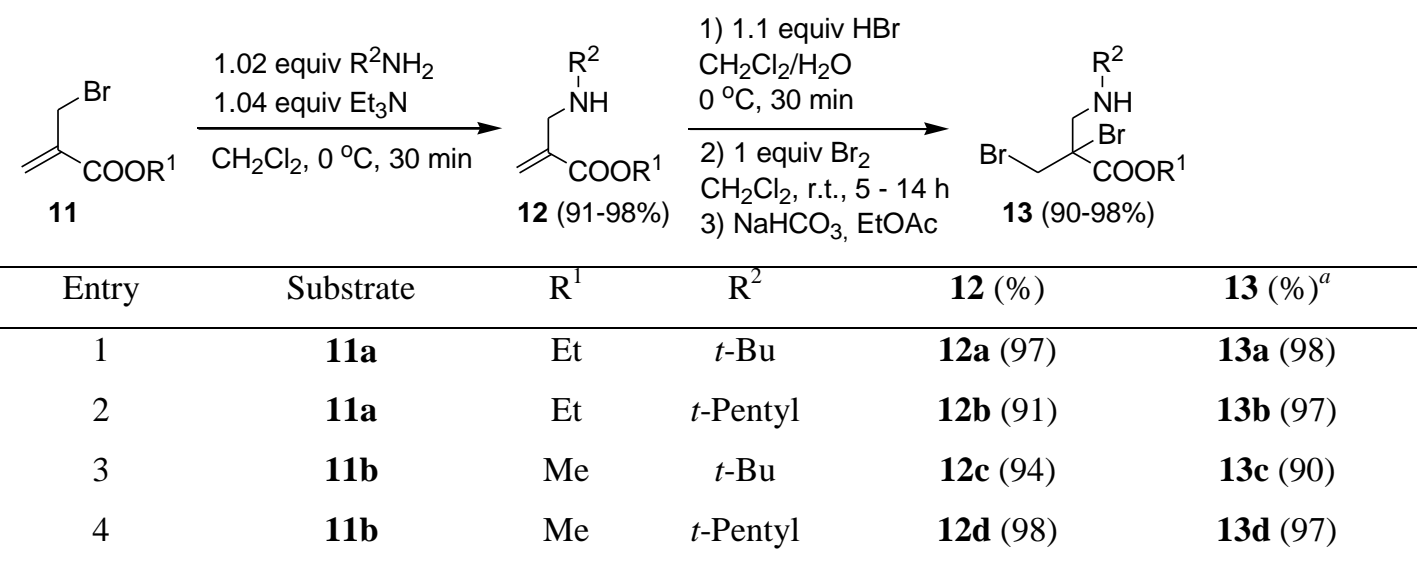

${ }^{a}$ Values between parentheses indicate yields of isolated products

With the targeted dibromo amino esters $\mathbf{1 3}$ in hand, their ring closure upon treatment under basic conditions to the desired aziridines $\mathbf{1 4}$ and/or azetidines $\mathbf{1 5}$ was investigated. After extensive screening of different bases, solvents, reaction temperatures and reaction times, it was found that a clean conversion of dibromo amines $\mathbf{1 3}$ to the aziridines $\mathbf{1 4}$ as major products together with the azetidines $\mathbf{1 5}$ as minor compounds could be achieved by using $\mathrm{K}_{2} \mathrm{CO}_{3}$ in acetonitrile at $60{ }^{\circ} \mathrm{C}$. However, the yields of the isolated aziridines 14 and azetidines 15 were low after purification by flash chromatography on silicagel as, apparently, these azaheterocycles partly decompose on column (Table 2, entry 1). To our satisfaction, good overall yields of the targeted isolated aziridines 14 (44-54\%) and azetidines 15 (24-33\%) were obtained via separation by preparative TLC up to $1 \mathrm{~g}$ scale (Table 2, entries 2-5).

It was envisioned that aziridines $\mathbf{1 4}$ are the kinetic cyclization products, while azetidines 15 result from a thermodynamical equilibration. The isomerization of 2-(halomethyl)aziridines to 3-haloazetidines, although only in some cases observable for 2-(chloromethyl)aziridines which lack a second substituent at the 2-position, was explained via the intermediacy of a bicyclic azonia[1.1.0]bicyclobutane I and eventually the corresponding carbenium ion II, followed by recombination with the initially expelled halide to give 3haloazetidines or 2-(halomethyl)aziridines (Gaertner 1970; Higgins and Kidd 1998). In order to experimentally prove this isomerization of aziridine 14 to azetidine 15, alkyl 2-(bromomethyl)aziridine-2-carboxylates 14 were heated under several conditions similar to the reaction conditions used in the preparation of azetidines $\mathbf{1 5}$ from acyclic dibromo amino esters $\mathbf{1 3}$ (Table 3). 
Table 2 Synthesis of alkyl aziridine-2-carboxylates 14 and alkyl azetidine-3-carboxylates 15

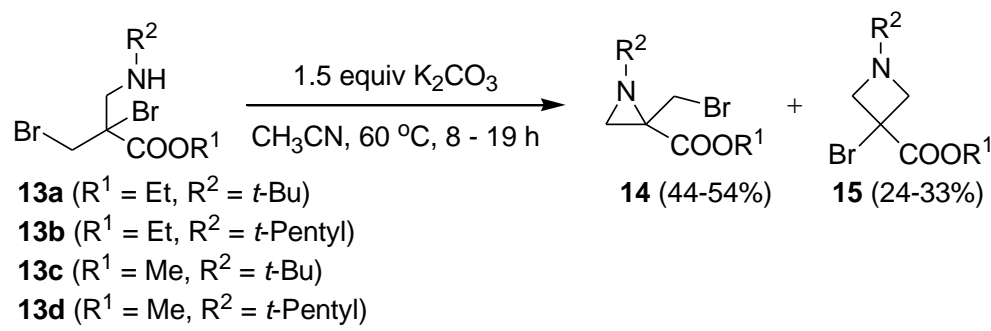

\begin{tabular}{cccc}
\hline Entry & Substrate & Reaction time $(\mathrm{h})$ & ${\text { Result }(\%)^{a, b}}^{\text {13a }}$ \\
\hline 1 & 13a & 19 & $\mathbf{1 4 a}(19)^{a}+\mathbf{1 5 a}(13)^{a}$ \\
2 & 13a & 19 & $\mathbf{1 4 a}(50)^{b}+\mathbf{1 5 a}(33)^{b}$ \\
3 & 13b & 12 & $\mathbf{1 4 b}(54)^{b}+\mathbf{1 5 b}(24)^{b}$ \\
4 & 13c & 8 & $\mathbf{1 4 c}(44)^{b}+\mathbf{1 5 c}(25)^{b}$ \\
5 & 13d & 18 & $\mathbf{1 4 d}(54)^{b}+\mathbf{1 5 d}(26)^{b}$
\end{tabular}

${ }^{a}$ Values between parentheses indicate yields of isolated products after purification by flash chromatography

${ }^{b}$ Values between parentheses indicate yields of isolated products after purification by preparative TLC

Table 3 Isomerization of 2-(bromomethyl)aziridines 14 to 3-bromoazetidines 15

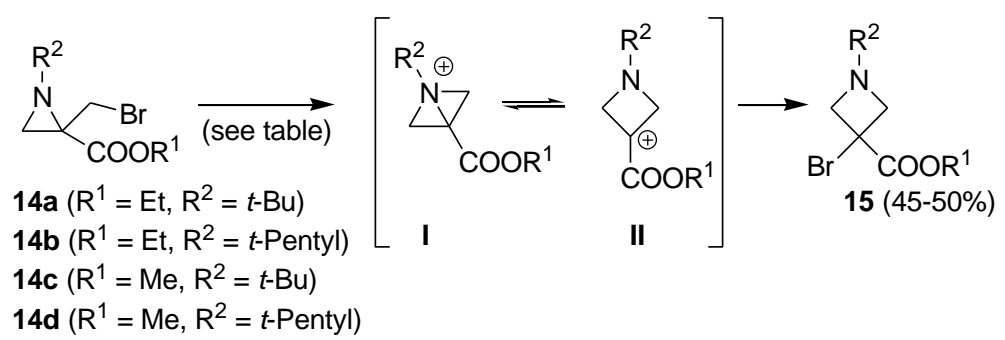

\begin{tabular}{cccc}
\hline Entry & Substrate & Reaction conditions & Result \\
\hline 1 & $\mathbf{1 4 a}$ & $\mathrm{CH}_{3} \mathrm{CN}, 60^{\circ} \mathrm{C}, 3 \mathrm{~h}$ & No reaction \\
2 & $\mathbf{1 4 a}$ & $\mathrm{CH}_{3} \mathrm{CN}, \Delta, 15 \mathrm{~h}$ & Decomposition \\
3 & $\mathbf{1 4 a}$ & 1 equiv $\mathrm{KBr}, \mathrm{CH}_{3} \mathrm{CN}, 60^{\circ} \mathrm{C}, 24 \mathrm{~h}$ & Decomposition \\
4 & $\mathbf{1 4 a}$ & $\mathrm{DMSO}, 55^{\circ} \mathrm{C}, 24 \mathrm{~h}$ & $\mathbf{1 4 a : 1 5 a}=2: 1^{a}$ \\
5 & $\mathbf{1 4 a}$ & $\mathrm{DMSO}, 55^{\circ} \mathrm{C}, 24 \mathrm{~h}+65^{\circ} \mathrm{C}, 6 \mathrm{~h}$ & $\mathbf{1 4 a}: \mathbf{1 5 a}=1: 2.8^{a}$ \\
6 & $\mathbf{1 4 a}$ & $\mathrm{DMSO}, 70^{\circ} \mathrm{C}, 18 \mathrm{~h}$ & $\mathbf{1 5 a}(45 \%)^{b}$ \\
7 & $\mathbf{1 4 b}$ & $\mathrm{DMSO}, 70^{\circ} \mathrm{C}, 45 \mathrm{~h}$ & $\mathbf{1 5 b}(45 \%)^{b}$ \\
8 & $\mathbf{1 4 c}$ & $\mathrm{DMSO}, 70^{\circ} \mathrm{C}, 5 \mathrm{~h}$ & $\mathbf{1 5 c}(45 \%)^{b}$ \\
9 & $\mathbf{1 4 d}$ & $\mathrm{DMSO}, 70^{\circ} \mathrm{C}, 5 \mathrm{~h}$ & $\mathbf{1 5 d}(50 \%)^{b}$ \\
10 & $\mathbf{1 4 a}$ & 1 equiv $\mathrm{KBr}, \mathrm{DMSO}, 70^{\circ} \mathrm{C}, 18 \mathrm{~h}$ & $\mathbf{1 5 a}(26 \%)^{b}$ \\
11 & $\mathbf{1 4 a}$ & $\mathrm{DMSO} / \mathrm{H}_{2} \mathrm{O} 1 / 1,70{ }^{\circ} \mathrm{C}, 48 \mathrm{~h}$ & Decomposition \\
12 & $\mathbf{1 4 a}$ & 1 equiv $\mathrm{KBr}, \mathrm{DMSO} / \mathrm{H}_{2} \mathrm{O} 1 / 1,70{ }^{\circ} \mathrm{C}, 48 \mathrm{~h}$ & Decomposition \\
\hline
\end{tabular}

\footnotetext{
${ }^{a}$ Ratio determined by ${ }^{1} \mathrm{H}$ NMR analysis

${ }^{b}$ Values between parentheses indicate yields of isolated products
} 
Heating of aziridine 14a in acetonitrile at $60{ }^{\circ} \mathrm{C}$ for $3 \mathrm{~h}$ gave no reaction (entry 1 ), while prolonged heating in acetonitrile at $60{ }^{\circ} \mathrm{C}$ in the presence of $\mathrm{KBr}$, which is formed in situ during the cyclization of 13, resulted in decomposition to unidentified compounds after $24 \mathrm{~h}$ (entry 3). Nevertheless, a satisfactory isomerization of aziridine 14a to azetidine 15a was observed upon heating in DMSO, giving a mixture of $\mathbf{1 4 a}$ and $\mathbf{1 5 a}$ in a $2: 1$ ratio $\left({ }^{1} \mathrm{H} \mathrm{NMR}\right.$ analysis) at 55 ${ }^{\circ} \mathrm{C}$ after $24 \mathrm{~h}$. The latter mixture further isomerized to $\mathbf{1 4 a}$ and $\mathbf{1 5 a}$ in a 1:2.8 ratio after additional heating at $65{ }^{\circ} \mathrm{C}$ for $6 \mathrm{~h}$. After raising the temperature to $70{ }^{\circ} \mathrm{C}$, full isomerization of aziridines 14a-d to azetidines 15a-d was obtained within 45 h with 45-50\% yield (Table 3, entries 6-9). Attempts to improve the outcome of the isomerization reaction by adding $\mathrm{KBr}$ resulted in a lower yield of $26 \%$ of azetidine 15a, while addition of water to the reaction (Table 3, entries 11-12) slowed down the isomerization and eventually led to total decomposition.

Introduction of new functional groups in alkyl aziridine-2-carboxylates $\mathbf{1 4}$ and alkyl azetidine-3-carboxylates $\mathbf{1 5}$ in particular, would highly enrich the chemistry of these important classes of $\alpha$ - and $\beta$-amino acid derivatives. Therefore, the potential of the synthesized alkyl 2-(bromomethyl)aziridine-2-carboxylates 14 and alkyl 3-bromoazetidine-3-carboxylates $\mathbf{1 5}$ for further functionalization via substitution reactions with $C$-, $S$-, $N$ - and $O$-nucleophiles was investigated (Tables 4 and 5). Treatment of alkyl 2-(bromomethyl)aziridine-2-carboxylates 14a-c with potassium cyanide in DMSO at $60{ }^{\circ} \mathrm{C}$ for $3-4 \mathrm{~h}$ uneventfully afforded the corresponding alkyl 2-(cyanomethyl)aziridine-2-carboxylates 16a-c in $47-57 \%$ yield (Table 4, entries 1-3). Few 2-(cyanomethyl)aziridines can be found in the literature (Gaertner 1970; Subbaraj et al. 1989; Antunes et al. 2007; Vedejs et al. 2003; Krasnova et al. 2005), and some have been used recently for the synthesis of biologically relevant $N$-(2-cyanocyclopropyl)benzimidates (D'hooghe et al. 2006a), 2-aminocyclopropanecarbonitriles (Mangelinckx et al. 2009), 4-amino-2butenenitriles (D'hooghe et al. 2007a, 2007b), 3,4-diaminobutanenitriles (D'hooghe et al. 2007a), and 2-aminopentanedinitriles (D'hooghe et al. 2008). Ethyl 2-(thiocyanomethyl)aziridine-2-carboxylates 17a-b were synthesized in moderate yield $(44-48 \%)$ utilizing potassium thiocyanate in DMF at $70{ }^{\circ} \mathrm{C}$ due to the formation of some unidentified reaction products which were present in the crude reaction mixture (Table 4, entries 4-5). Only one report on the synthesis of 
2-(thiocyanomethyl)aziridines, which were used for an intramolecular cyclisation to 2-iminothiazolidines, has been made (D'hooghe et al. 2005b).

Table 4 Reactions of alkyl 2-(bromomethyl)aziridines 14 with different nucleophiles

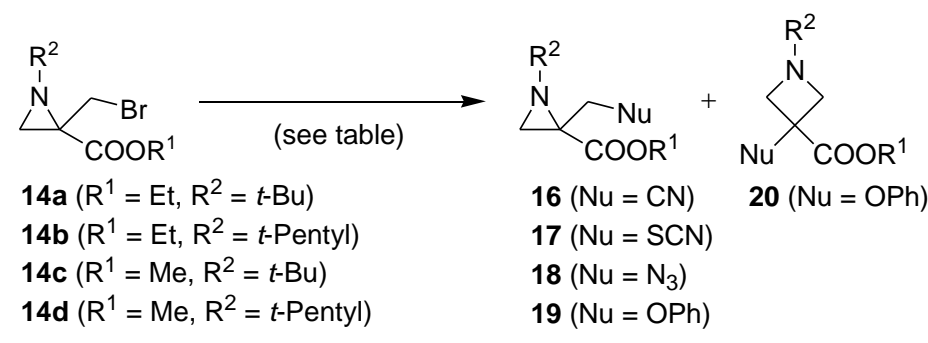

\begin{tabular}{|c|c|c|c|}
\hline Entry & Substrate & Reaction conditions & Result $(\%)^{a}$ \\
\hline 1 & $14 \mathbf{a}$ & 1 equiv $\mathrm{KCN}, \mathrm{DMSO}, 60^{\circ} \mathrm{C}, 3 \mathrm{~h}$ & 16a (47) \\
\hline 2 & $14 \mathrm{~b}$ & 1 equiv $\mathrm{KCN}, \mathrm{DMSO}, 60^{\circ} \mathrm{C}, 4 \mathrm{~h}$ & 16b (54) \\
\hline 3 & $14 c$ & 1 equiv $\mathrm{KCN}, \mathrm{DMSO}, 60^{\circ} \mathrm{C}, 4 \mathrm{~h}$ & 16c (57) \\
\hline 4 & $14 a$ & 2 equiv $\mathrm{KSCN}, \mathrm{DMF}, 70^{\circ} \mathrm{C}, 2 \mathrm{~h}$ & $\mathbf{1 7 a}(48)$ \\
\hline 5 & $14 b$ & 2 equiv $\mathrm{KSCN}, \mathrm{DMF}, 70^{\circ} \mathrm{C}, 6 \mathrm{~h}$ & 17b (44) \\
\hline 6 & $14 a$ & 2 equiv $\mathrm{NaN}_{3}, \mathrm{DMSO}, 60^{\circ} \mathrm{C}, 1.5 \mathrm{~h}$ & 18a $(58)$ \\
\hline 7 & $14 \mathrm{~b}$ & 2 equiv $\mathrm{NaN}_{3}, \mathrm{DMSO}, 60^{\circ} \mathrm{C}, 2 \mathrm{~h}$ & $\mathbf{1 8 b}(93)$ \\
\hline 8 & $14 c$ & 2 equiv $\mathrm{NaN}_{3}, \mathrm{DMSO}, 60^{\circ} \mathrm{C}, 3 \mathrm{~h}$ & $18 c(91)$ \\
\hline 9 & 14d & 2 equiv $\mathrm{NaN}_{3}, \mathrm{DMSO}, 60^{\circ} \mathrm{C}, 3 \mathrm{~h}$ & 18d (64) \\
\hline 10 & $14 a$ & $\begin{array}{c}2.2 \text { equiv } \mathrm{PhOH}, 5 \text { equiv } \mathrm{K}_{2} \mathrm{CO}_{3} \\
\text { acetone/DMF } 1 / 1, \Delta, 125 \mathrm{~h}\end{array}$ & 19a $(10)$ \\
\hline 11 & $14 \mathrm{~b}$ & $\begin{array}{c}2.2 \text { equiv } \mathrm{PhOH}, 5 \text { equiv } \mathrm{K}_{2} \mathrm{CO}_{3} \\
\text { acetone/DMF } 1 / 1, \Delta, 160 \mathrm{~h}\end{array}$ & - \\
\hline 12 & $14 a$ & $\begin{array}{c}2 \text { equiv } \mathrm{PhOH}, 4 \text { equiv } \mathrm{K}_{2} \mathrm{CO}_{3} \\
\text { DMSO, } 60^{\circ} \mathrm{C}, 8 \mathrm{~h}\end{array}$ & $19 \mathbf{a}(60)+20 a(5)$ \\
\hline 13 & $14 b$ & $\begin{array}{c}2 \text { equiv } \mathrm{PhOH}, 4 \text { equiv } \mathrm{K}_{2} \mathrm{CO}_{3} \\
\text { DMSO, } 60^{\circ} \mathrm{C}, 5 \mathrm{~h}\end{array}$ & $19 b(34)+20 b(4)$ \\
\hline 14 & $14 b$ & $\begin{array}{c}3 \text { equiv } \mathrm{PhOH}, 6 \text { equiv } \mathrm{K}_{2} \mathrm{CO}_{3} \\
\text { DMSO, } 60{ }^{\circ} \mathrm{C}, 16 \mathrm{~h}\end{array}$ & $19 b(67)$ \\
\hline
\end{tabular}

${ }^{a}$ Values between parentheses indicate yields of isolated products

Alkyl 2-(azidomethyl)aziridine-2-carboxylates 18a-d were prepared in good to excellent yield by reaction with sodium azide in DMSO at $60{ }^{\circ} \mathrm{C}$ (Table 4 , entries 6-9). 2-(Azidomethyl)aziridines are also scarcely reported in the literature (Jamookeeah et al. 2008; Han et al. 2008; D'hooghe et al. 2005a). Methyl (2R,3R)-1-benzyl-3-(azidomethyl)-2-aziridinecarboxylate has been used in routes towards the synthesis of aziridinomitosenes as antitumor antibiotics (Shaw et al. 1985). Upon reaction of ethyl 2-(bromomethyl)aziridine-2-carboxylates 14a-b 
with phenol and $\mathrm{K}_{2} \mathrm{CO}_{3}$ in a mixture of acetone and DMF (1/1) under reflux, no satisfactory results were obtained even after prolonged reaction time (Table 4 , entries 10-11). However, treating ethyl 2-(bromomethyl)aziridine-2-carboxylates 14a-b with phenol and $\mathrm{K}_{2} \mathrm{CO}_{3}$ in DMSO at $60{ }^{\circ} \mathrm{C}$ for 5 to 16 hours afforded ethyl 1-tert-butyl-2-(phenoxymethyl)aziridine-2-carboxylate 19a and ethyl 1-tertpentyl-2-(phenoxymethyl)aziridine-2-carboxylate 19b in $60-67 \%$ yield (Table 4 , entries 12-14). Noteworthy, besides the aziridines 19 as major compounds, the rearranged compounds, i.e. ethyl 1-tert-butyl-3-phenoxyazetidine-3-carboxylate 20a and ethyl 1-tert-pentyl-3-phenoxyazetidine-3-carboxylate 20b, were isolated in small amounts (4-5\%) from this reaction. 2-(Aryloxymethyl)aziridines have recently been used for the synthesis of biologically relevant 2-amino-1-aryloxy-3methoxypropanes (D'hooghe et al. 2006b), and for the synthesis of $1 H$-tetrazole1-alkanenitriles as potent orally bioavailable growth hormone secretagogues (Hernández et al. 2008).

In analogy with the reactions of aziridines 14, treatment of the 3-bromoazetidines 15 with potassium cyanide, potassium thiocyanate, sodium azide and potassium phenoxide in DMSO at $60{ }^{\circ} \mathrm{C}$ for 3 to 4 hours afforded the corresponding 3cyano-, 3-thiocyano-, 3-azido- and 3-phenoxyazetidine-3-carboxylic esters 20-23 in 36-98\% yield (Table 5). 3-Substituted azetidines are frequently used in structure-activity relationship (SAR) studies for the preparation of libraries of bioactive compounds, e.g. bronchodilating and anti-inflammatory drugs (Provins et al. 2007), antibacterial agents (Murphy et al. 2007), and antidepressant agents (Melloni et al. 1979).

In order to extend the potential applicability of the synthesized constrained amino acid derivatives as building blocks for the synthesis of foldamers, the deprotection of several amino acid derivatives was studied as well. Using simple hydrogenolysis, ethyl 1-tert-pentyl-2-(azidomethyl)aziridine-2-carboxylate $\mathbf{1 8 b}$ and 1-tert-butyl-3-azidoazetidine-3-carboxylate 23a were converted into the corresponding ethyl 2-(aminomethyl)aziridine-2-carboxylate $\mathbf{2 4 b}$ and 3aminoazetidine-3-carboxylate 26a (Scheme 1 and 2). 
Table 5 Reactions of alkyl 3-bromoazetidines 15 with different nucleophiles

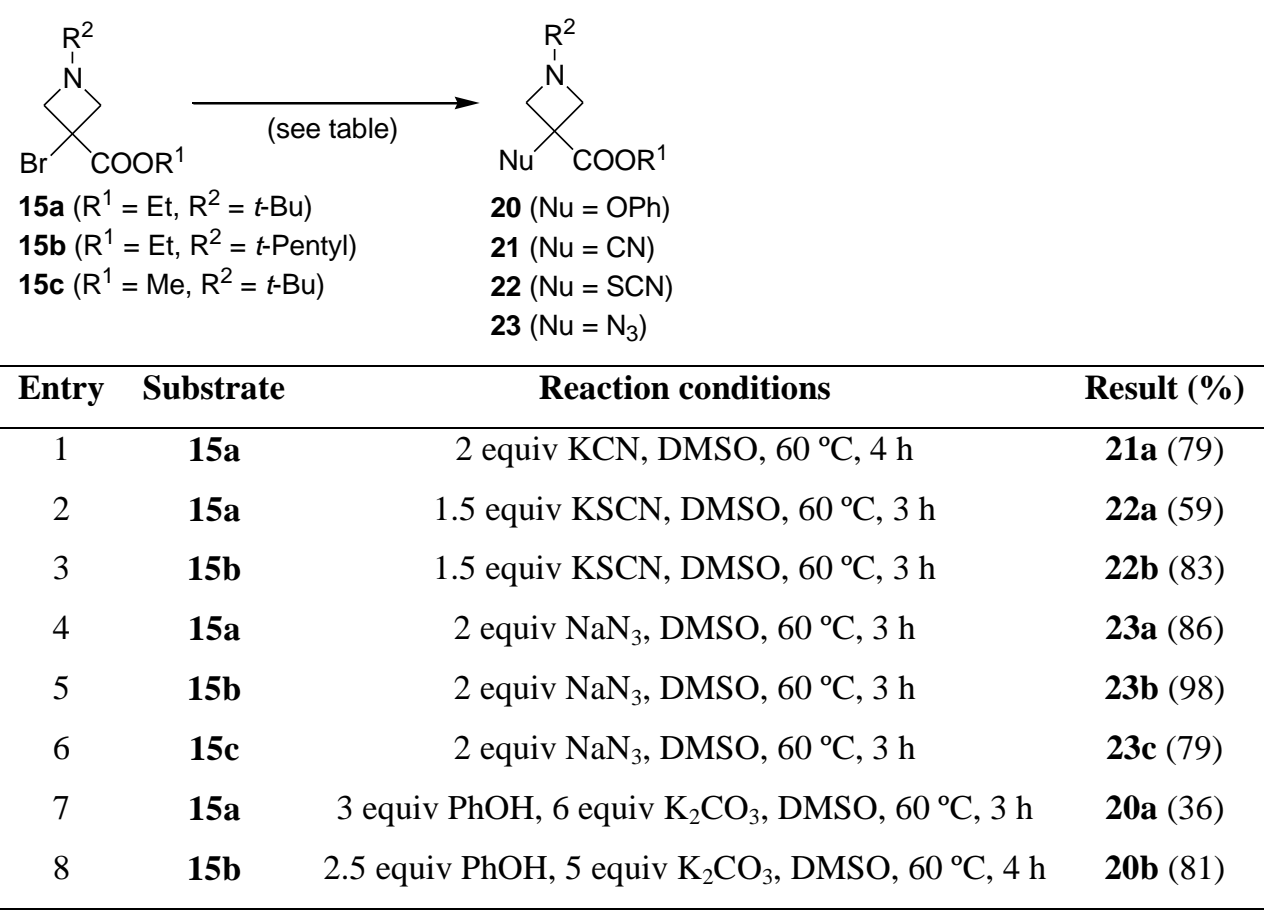

Noteworthy, the ring opening of 2-(aminomethyl)aziridine templates with thioacid peptides has recently been developed as a method for chemoselective peptidomimetic ligation (Assem et al. 2010). Furthermore, 2(azidomethyl)aziridines $\mathbf{1 8}$ could be directly transformed into the corresponding $\mathrm{N}$-Boc-protected 2-(aminomethyl)aziridines $\mathbf{2 5}$ by hydrogenation in the presence of di-tert-butyl dicarbonate (Scheme 1). All efforts to saponify 2(azidomethyl)aziridine-2-carboxylic esters via basic hydrolysis (aq. $\mathrm{Na}_{2} \mathrm{CO}_{3}$ or aq. $\mathrm{LiOH}$ ) failed, resulting in either no reaction or formation of complex reaction mixtures. However, simple hydrolysis of ethyl 3-azidoazetidine-3-carboxylates 23a,b to the corresponding 3-azidoazetidine-3-carboxylic acids 27 was achieved upon reaction with $2 \mathrm{~N}$ aqueous $\mathrm{NaOH}$ solution in methanol at room temperature for $3 \mathrm{~h}$ (Scheme 2). Further hydrogenolysis of 3-azidoazetidine-3-carboxylic acids 27 afforded 3-aminoazetidine-3-carboxylic acids $\mathbf{2 8}$. Besides their potential use as foldameric building blocks, the elaborated 3-aminoazetidine-3-carboxylates 26-28 can be important for the synthesis of series of active compounds used in SAR, as exemplified for EGF receptor tyrosine kinase inhibitors (Hennequin et al. 2006), $\mathrm{CB}_{1}$ receptor antagonists (Brandt et al. 2009), and modulators of the NMDA receptor complex (Kozikowski and Fauq 1991). 


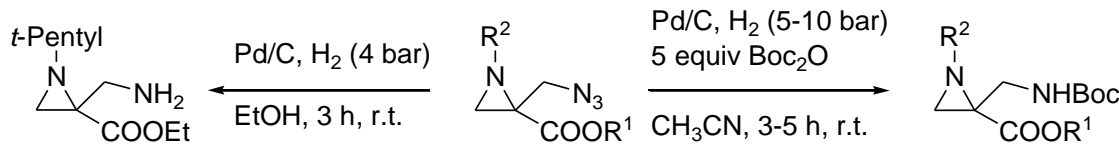

$$
\begin{aligned}
& \text { 24b (68\%) } \\
& 18
\end{aligned}
$$

\section{Scheme 1}
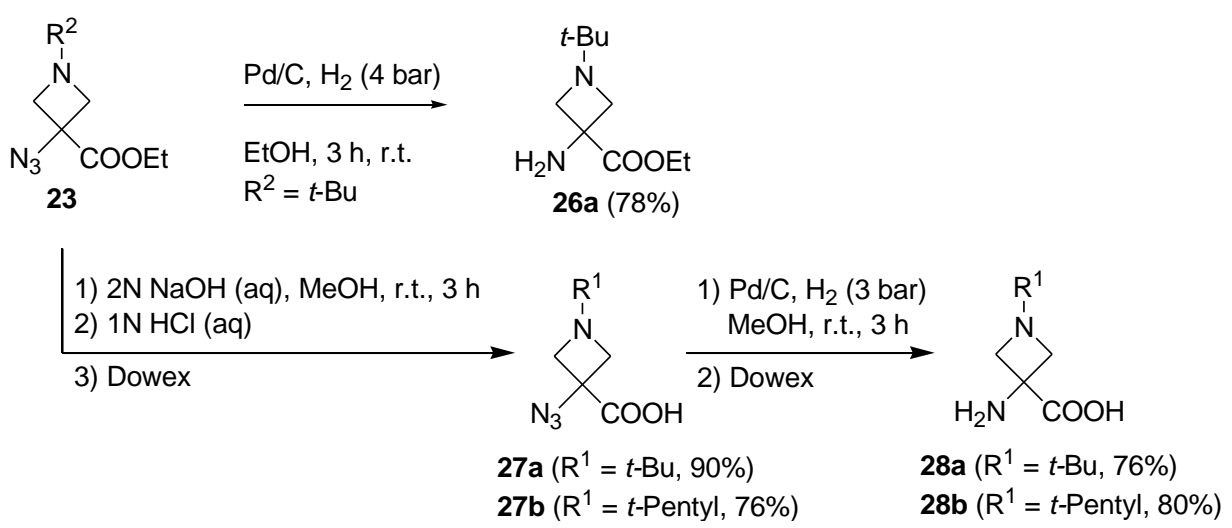

Scheme 2

In conclusion, a short synthesis of alkyl 2-(bromomethyl)aziridine-2-carboxylates and alkyl 3-bromoazetidine-3-carboxylates was developed involving amination, bromination, and base-induced cyclization of alkyl 2-(bromomethyl)acrylates. The aziridines are the kinetically favored cyclization products and could be transformed into 3-bromoazetidine-3-carboxylic acid derivatives via thermal isomerization. The new small ring azaheterocyclic $\alpha$ - and $\beta$-amino acid derivatives proved to be excellent building blocks for the synthesis of different substituted aziridines and azetidines through nucleophilic substitution of the bromide by different carbon, sulfur, oxygen and nitrogen nucleophiles in good to high yields. Elaboration of the amino and carboxyl group made new conformationally constrained functionalized $\alpha$ - and $\beta$-amino acids synthetically accessible for potential application in foldamer research.

Acknowledgements The authors are indebted to the Research Foundation - Flanders (FWO Flanders) and Ghent University (BOF) for financial support.

\section{References}


Abbaspour Tehrani K, Nguyen Van T, Karikomi M, Rottiers M, De Kimpe N (2002) Electron transfer induced ring opening of 2-(bromomethyl)aziridines by magnesium in methanol. Tetrahedron 58: 7145-7152.

Abele S, Seiler P, Seebach D (1999) Synthesis, crystal structures, and modelling of $\beta$ oligopeptides consisting of 1-(aminomethyl)cyclopropanecarboxylic acid: ribbon-type arrangement of eight-membered H-bonded rings. Helv. Chim. Acta 82: 1559-1571.

Antunes AMM, Bonifacio VDB, Nascimento SCC, Lobo AM, Branco PS, Prabhakar S (2007) Palladium(II) mediated aziridination of olefins with bromamine-T as the nitrogen source: scope and mechanism. Tetrahedron 63: 7009-7017.

Assem N, Natarajan A, Yudin AK (2010) Chemoselective peptidomimetic ligation using thioacid peptides and aziridine templates. J. Am. Chem. Soc. 132: 10986-10987.

Baraki H, Habaue S, Okamoto Y (1999) Stereospecific anionic polymerization and novel hydrogen-transfer polymerization of $\alpha$-(aminomethyl)acrylates having unprotected amino group. Polym. J. 31: 1260-1266.

Brandt TA, Caron S, Damon DB, DiBrino J, Ghosh A, Griffith DA, Kedia S, Ragan JA, Rose PR, Vanderplas BC, Wei L (2009) Development of two synthetic routes to CE-178,253, a CB antagonist for the treatment of obesity. Tetrahedron 65: 3292-3304.

Cardillo G, Gentilucci L, Tolomelli A (2003) Aziridines and oxazolines: valuable intermediates in the synthesis of unusual amino acids. Aldrichim. Acta 36: 39-50.

Cardillo G, Gentilucci L, Tolomelli A (2006) Unusual Amino Acids: Synthesis and introduction into naturally occurring peptides and biologically active analogues. Mini-Rev. Med. Chem. 6: 293-304.

Cativiela C, Ordóñez M (2009) Recent progress on the stereoselective synthesis of cyclic quaternary $\alpha$-amino acids. Tetrahedron: Asymm. 20: 1-63.

Cheng RP, Gellman SH, DeGrado WF (2001) $\beta$-Peptides: from structure to function. Chem. Rev. 101: 3219-3232.

Cowell SM, Lee YS, Cain JP, Hruby VJ (2004) Exploring Ramachandran and Chi space: conformationally constrained amino acids and peptides in the design of bioactive polypeptide ligands. Curr. Med. Chem. 11: 2785-2798.

D’hooghe M, Mangelinckx S, Persyn E, Van Brabandt W, De Kimpe N (2006a) Synthesis of 1arylmethyl-2-(cyanomethyl)aziridines and their ring transformation into methyl $\mathrm{N}$-(2cyanocyclopropyl)benzimidates. J. Org. Chem. 71: 4232-4236.

D'hooghe M, Rottiers M, Kerkaert I, De Kimpe N (2005a) Ring opening of 2-(bromomethyl)-1sulfonylaziridines towards 1,3-heteroatom substituted 2-aminopropane derivatives. Tetrahedron 61: 8746-8751.

D'hooghe M, Van Nieuwenhove A, Van Brabandt W, Rottiers M, De Kimpe N (2008) Novel synthesis of 2-aminopentanedinitriles from 2-(bromomethyl)aziridines and their transformation into 2-imino-5-methoxypyrrolidines and 5-methoxypyrrolidin-2-ones. Tetrahedron 64: 1064-1070.

D'hooghe M, Van Speybroeck V, Van Nieuwenhove A, Waroquier M, De Kimpe N (2007a) Novel synthesis of 3,4-diaminobutanenitriles and 4-amino-2-butenenitriles from 2- 
(cyanomethyl)aziridines through intermediate aziridinium salts: an experimental and theoretical approach. J. Org. Chem. 72: 4733-4740.

D'hooghe M, Vervisch K, Van Nieuwenhove A, De Kimpe N (2007b) Ring opening of 2(cyanomethyl)aziridines by acid chlorides: synthesis of novel 4-amino-2-butenenitrile derivatives through intermediate aziridinium salts. Tetrahedron Lett. 48: 1771-1774.

D'hooghe M, Waterinckx A, De Kimpe, N (2005b) A novel entry toward 2-imino-1,3thiazolidines and 2-imino-1,3-thiazolines by ring transformation of 2(thiocyanomethyl)aziridines. J. Org. Chem. 70: 227-232.

D'hooghe M, Waterinckx A, Vanlangendonck T, De Kimpe N (2006b) A new approach towards 2-amino-1-aryloxy-3-methoxypropanes from 1-arylmethyl-2-(bromomethyl)aziridines. Tetrahedron 62: 2295-2303.

De Kimpe N, De Smaele D, Szakonyi Z (1997) A new synthesis of 2-methyleneaziridines. J. Org. Chem. 62: 2448-2452.

De Smaele D, Bogaert P, De Kimpe N (1998) Synthesis of pyrrolizidines by cascade reactions of $N$-alkenylaziridinylmethyl radicals. Tetrahedron Lett. 39: 9797-9800.

De Smaele D, Dejaegher Y, Duvey G, De Kimpe N (2001) A new entry towards the synthesis of 1-substituted 3-azetidinones. Tetrahedron Lett. 42: 2373-2375.

Devlin BRJ (1981) Azetidine derivatives, pollen suppressing compositions containing them, their use in sterilizing male anthers in plants and a method of producing F1 hybrid seed. Eur. Pat. Appl. EP 29265.

Frigola J, Pares J, Corbera J, Vano D, Merce R, Torrens A, Mas J, Valenti E (1993) 7Azetidinylquinolones as antibacterial agents. Synthesis and structure-activity relationships. J. Med. Chem. 36: 801-810.

Fülöp F, Martinek TA, Tóth G (2006) Application of alicyclic $\beta$-amino acids in peptide chemistry. Chem. Soc. Rev. 35: 323-334.

Gaertner VR (1970) Reactions of nucleophiles with 1-tert-butyl-3-chloroazetidine and 1-tertbutyl-2-chloromethylaziridine. J. Org. Chem. 35: 3952-3959.

Gelmi ML, Pocar D (2003) Heterosubstituted carbocyclic $\alpha$-amino acids. A review. Org. Prep. Proced. Int. 35: 141-205.

Gensler WJ (1948) The benzenesulfonyl derivatives of 1-amino-2,3-dibromopropane and 2-amino1,3-dibromopropane. J. Am. Chem. Soc. 70: 1843-1846.

Habaue S, Baraki H, Okamoto Y (1997) Stereospecific anionic polymerization of $\alpha$-(N-tert-butyl aminomethyl)acrylate having unprotected amino group. Polym. J. 29: 872-874.

Han SM, Ma SH, Ha HJ, Lee WK (2008) Nucleophilic substitution of (sulfonyloxymethyl)aziridines: an asymmetric synthesis of both isomers of mexiletine. Tetrahedron 64: 11110-11114.

Hayashi K, Ikee Y, Goto S, Shiro M, Nagao Y (2004) Mechanistic considerations for the consecutive cyclization of 2,3-dibromopropylamine hydrobromide giving a strained molecule, 1-azabicyclo[1.1.0]butane. Chem. Pharm. Bull. 52: 89-94.

Hennequin LFA, Ballard P, Boyle FT, Delouvrie B, Ellston RPA, Halsall CT, Harris CS, Hudson K, Kendrew J, Pease JE, Ross HS, Smith P, Vincent JL (2006) Novel 4-anilinoquinazolines 
with C-6 carbon-linked side chains: synthesis and structure-activity relationship of a series of potent, orally active, EGF receptor tyrosine kinase inhibitors. Bioorg. Med. Chem. Lett. 16: 2672-2676.

Hernández AS, Swartz SG, Slusarchyk D, Yan M, Seethala RK, Sleph P, Grover G, Dickinson K, Giupponi L, Harper TW, Humphreys WG, Longhi DA, Flynn N, Murphy BJ, Gordon DA, Biller SA, Robl JA, Tino JA (2008) Optimization of $1 H$-tetrazole-1-alkanenitriles as potent orally bioavailable growth hormone secretagogues. Bioorg. Med. Chem. Lett. 18: 2067-2072.

Higgins RH, Kidd B (1998) An ab initio study of 1-azabicyclo[1.1.0]butyl and isomeric cations. J. Phys. Org. Chem. 11: 763-773.

Hughes AB (Ed) (2009a) Amino acids, peptides and proteins in organic chemistry, volume 1 origins and synthesis of amino Acids. Wiley-VCH, Weinheim.

Hughes B (2009b) 2008 FDA drug approvals. Nat. Rev. Drug Discov. 8: 93-96.

Ide ND, Galonić DP, van der Donk WA, Gin DY (2005) Conjugation of selenols with aziridine-2carboxylic acid-containing peptides. Synlett: 2011-2014.

Jamookeeah CE, Beadle CD, Jackson RFW, Harrity JPA (2008) Investigation of a flexible enantiospecific approach to aziridines. J. Org. Chem. 73: 1128-1130.

Kiss L, Fülöp F (2010) Selective syntheses of functionalized cyclic $\beta$-amino acids via transformation of the ring C-C double bonds. Synlett: 1302-1314.

Komarov IV, Grigorenko AO, Turov AV, Khilya VP (2004) Conformationally rigid cyclic $\alpha$ amino acids in the design of peptidomimetics, peptide models and biologically active compounds. Russ. Chem. Rev. 73: 785-810.

Kozikowski AP, Fauq AH (1991) Synthesis of novel four-membered ring amino acids as modulators of the $N$-methyl-D-aspartate (NMDA) receptor complex. Synlett: 783-784.

Krasnova LB, Hili RM, Chernoloz OV, Yudin AK (2005) Phenyliodine(III) diacetate as a mild oxidant for aziridination of olefins and imination of sulfoxides with $\mathrm{N}$-aminophthalimide. ARKIVOC iv: 26-38.

Kuhl A, Hahn MG, Dumić M, Mittendorf J (2005) Alicyclic $\beta$-amino acids in medicinal chemistry. Amino Acids 29: 89-100.

Lee WK, Ha HJ (2003) Highlights of the chemistry of enantiomerically pure aziridine-2carboxylates. Aldrichim. Acta 36: 57-63.

Lucarini S, Tomasini C (2001) Synthesis of oligomers of trans-(4S,5R)-4-carboxybenzyl 5-methyl oxazolidin-2-one: an approach to new foldamers. J. Org. Chem. 66: 727-732.

Maity P, König B (2008) Enantio- and diastereoselective syntheses of cyclic $\mathrm{C}^{\alpha}$-tetrasubstituted $\alpha$ amino acids and their use to induce stable conformations in short peptides. Biopolymers (Pept. Sci.) 90: 8-27.

Mangelinckx S, D'hooghe M, Peeters S, De Kimpe N (2009) Synthesis of 2[(arylmethylene)amino]cyclopropanecarbonitriles via a two-step ring transformation of 2(cyanomethyl)aziridines. Synthesis: 1105-1112.

Mangelinckx S, Žukauskaitė A, Buinauskaitė V, Šačkus A, De Kimpe N (2008) Synthesis of alkyl 2-(bromomethyl)aziridine-2-carboxylates and alkyl 3-bromoazetidine-3-carboxylates as amino acid building blocks. Tetrahedron Lett. 49: 6896-6900. 
Mazzini C, Lebreton J, Alphand V, Furstoss R (1997) Enantiodivergent chemoenzymatic synthesis of $(R)$ - and $(S)$ - $\beta$-proline in high optical purity. J. Org. Chem. 62: 5215-5218.

Melloni P, Della Torre A, Meroni M, Ambrosini A, Rossi AC (1979) Azetidine derivatives of tricyclic antidepressant agents. J. Med. Chem. 22: 183-191.

Miller JA, Nguyen ST (2005) The enantioselective synthesis of conformationally constrained cyclic $\beta$-amino acids. Mini-Rev. Org. Chem. 2: 39-45.

Miller RA, Lang F, Marcune B, Zewge D, Song ZJ, Karady S (2003) A practical process for the preparation of azetidine-3-carboxylic acid. Synth. Commun. 33: 3347-3353.

Morieux P, Stables JP, Kohn H (2008) Synthesis and anticonvulsant activities of $N$-benzyl (2R)-2acetamido-3-oxysubstituted propionamide derivatives. Bioorg. Med. Chem. 16: 8968-8975.

Murphy ST, Case HL, Ellsworth E, Hagen S, Huband M, Joannides T, Limberakis C, Marotti KR, Ottolini AM, Rauckhorst M, Starr J, Stier M, Taylor C, Zhu T, Blaser A, Denny WA, Lu GL, Smaill JB, Rivault F (2007) The synthesis and biological evaluation of novel series of nitrilecontaining fluoroquinolones as antibacterial agents. Bioorg. Med. Chem. Lett. 17: 21502155 .

Orr AF, Clifford DR (1984) Azetidine derivatives suitable for inducing male sterility in plants. Brit. UK Pat. Appl. GB 2140003.

Park KH, Kurth MJ (2002) Cyclic amino acid derivatives. Tetrahedron 58: 8629-8659.

Pillai B, Cherney MM, Diaper CM, Sutherland A, Blanchard JS, Vederas JC, James MNG (2006) Structural insights into stereochemical inversion by diaminopimelate epimerase: an antibacterial drug target. Proc. Natl Acad. Sci. USA 103: 8668-8673.

Pillai B, Moorthie VA, van Belkum MJ, Marcus SL, Cherney MM, Diaper CM, Vederas JC, James MNG (2009) Crystal structure of diaminopimelate epimerase from Arabidopsis thaliana, an amino acid racemase critical for L-lysine biosynthesis. J. Mol. Biol. 385: 580594.

Provins L, Christophe B, Danhaive P, Dulieu J, Gillard M, Quéré L, Stebbins K (2007) Dual M antagonists-PDE4 inhibitors. Part 2: Synthesis and SAR of 3-substituted azetidinyl derivatives. Bioorg. Med. Chem. Lett. 17: 3077-3080.

Robin S, Rousseau G (2000) Preparation of azetidines by 4-endo trig cyclizations of $N$-cinnpentyl tosylamides. Eur. J. Org. Chem.: 3007-3011.

Robin S, Rousseau G (2002) Formation of four-membered heterocycles through electrophilic heteroatom cyclization. Eur. J. Org. Chem.: 3099-3114.

Seebach D, Gardiner J (2008) $\beta$-Peptidic peptidomimetics. Acc. Chem. Res. 41: 1366-1375.

Shaw KJ, Luly JR, Rapoport H (1985) Routes to mitomycins. Chirospecific synthesis of aziridinomitosenes. J. Org. Chem. 50: 4515-4523.

Soloshonok VA, Sorochinsky AE (2010) Practical methods for the synthesis of symmetrically $\alpha, \alpha$ disubstituted $\alpha$-amino acids. Synthesis: 2319-2344.

Subbaraj A, Rao OS, Lwowski W (1989) Functionalization of olefins by alkoximidoylnitrenes. J. Org. Chem. 54: 3945-3952.

Toniolo C, Formaggio F, Kaptein B, Broxterman QB (2006) You Are Sitting on a Gold Mine! Synlett: $1295-1310$. 
Van Brabandt W, Mangelinckx S, D'hooghe M, Van Driessche B, De Kimpe N (2009) Synthesis and reactivity of 3-haloazetidines and 3-sulfonyloxyazetidines: a review. Curr. Org. Chem. 13: 829-853 (and references therein).

Van Driessche B, Van Brabandt W, D’hooghe M, Dejaegher Y, De Kimpe N (2006) Synthesis and reactivity of trans-2-aryl-3-chloroazetidines. Tetrahedron 62: 6882-6892 (and references therein).

Vedejs E, Naidu BN, Klapars A, Warner DL, Li, VS, Na Y, Kohn H (2003) Synthetic enantiopure aziridinomitosenes: preparation, reactivity, and DNA alkylation studies. J. Am. Chem. Soc. 125: 15796-15806.

Verbrugge PA, De Waal J (1989) Process for preparation of azetidine-3-carboxylic acid, a gametocide. US Patent 4812581.

Vervisch K, D’hooghe M, Törnroos KW, De Kimpe N (2010) Synthesis of stereodefined piperidines from aziridines and their transformation into conformationally constrained amino acids, amino alcohols and 2,7-diazabicyclo[3.3.1]nonanes. J. Org. Chem. 75: 7734-7744.

Vicik R, Busemann M, Baumann K, Schirmeister T (2006) Inhibitors of cysteine proteases. Curr. Top. Med. Chem. 6: 331-353.

Zhang XH, Takagi H, Widholm JM (2004) Expression of a novel yeast gene that detoxifies the proline analog azetidine-2-carboxylate confers resistance during tobacco seed germination, callus and shoot formation. Plant Cell Rep. 22: 615-622. 